\title{
The Value of Information in Risk-Sharing Environments with Unawareness*
}

\author{
Spyros Galanis ${ }^{\dagger}$
}

March 18, 2016

\begin{abstract}
The value of information is examined in a risk-sharing environment with unawareness and complete markets. Information and awareness are symmetric among agents, who have a clear understanding of their actions and deterministic payoffs. We show with examples that public information can make some agents strictly better off at the expense of others, contrasting the standard results of Hirshleifer [1971] and Schlee [2001] that the value of public information is negative for all when risk averse agents are fully insured. We identify the source of this problem to be that, as awareness varies across states, it creates an awareness signal that the agents misunderstand and treat asymmetrically. As a result, risk-sharing opportunities that are available when this signal is not used, vanish when it is used. We identify a property, Conditional Independence, which we show is sufficient for the value of public information to be negative for all.
\end{abstract}

JEL-Classifications: C70, D53, D80, D82.

Keywords: unawareness, value of information, risk-sharing, uncertainty, knowledge, bounded perception, awareness.

\section{Introduction}

In standard neoclassical economies with complete markets, where symmetrically informed agents trade state-contingent claims under uncertainty, Hirshleifer [1971] shows that providing public information may hurt all market participants, as it reduces the opportunities for risk-sharing. To provide an example, consider an economy with two risk averse agents and two, equally likely, states of the world, $a_{h}$ (the level of the assets of a specific company is high) and $a_{l}$ (the level of the assets is low). The aggregate endowment for the economy is 1, constant across the two states. Agent 1 has all of the endowment at $a_{h}$ and nothing at $a_{l}$. If the agents do not receive any information before agreeing to trade, the unique equilibrium allocation is to consume $1 / 2$ in both states. Suppose now that public information is available, enabling the agents to know which state has occurred. Then there is no trade, as each agent consumes her endowment at each

${ }^{*}$ I am grateful for their useful comments to Paulo Barelli, Piero Gottardi, Larry G. Epstein, Martin Meier, Herakles Polemarchakis, David Rahman, Marzena Rostek, Fernando Vega-Redondo, Michael Vlassopoulos, Marek Weretka, Xiaojian Zhao, two referees and seminar participants at the European University Institute, the University of Southampton, the Summer in Birmingham workshop and the International Workshop on Unawareness, University of Queensland.

${ }^{\dagger}$ Department of Economics, School of Social Sciences, University of Southampton, Southampton, UK, s.galanis@soton.ac.uk. 
state. From an ex ante perspective, both agents are better off from not having this information, as they bear more risk from consuming 0 in one state and 1 in the other.

Schlee [2001] generalises this result, showing that the value of public information is weakly negative for all risk averse agents, in environments where there is no aggregate uncertainty, or there are risk neutral agents who fully insure the risk averse ones (e.g. competitive insurance markets with risk neutral insurers). ${ }^{1}$ This means that market participants would unanimously reject the release of public information.

In practice, however, the debate on whether it is beneficial to disclose market related information is far from being settled. ${ }^{2}$ For instance, private banks tend not to report the market value of all of their assets, whereas central banks are not required to reveal the identities of the institutions that use their emergency lending facilities. On the other hand, there is a series of "mark-to-market" legislations that aim to disclose information, adopted in 1994 (FAS 115) and 2007 (FAS 157) in the US.

In this paper we show that with unawareness, the release of public information could benefit some market participants, at the expense of others, even in a restrictive environment with complete markets, no aggregate uncertainty, common priors and risk aversion. Such an observation could explain why some are in favour of disclosing information, whereas others are not. It could also rationalize (as we show in Section 3) why some very sophisticated (and more aware) market participants (e.g. hedge fund managers) may even want to disclose some of their private information and awareness to their unsophisticated trading partners in order to take advantage of them.

The market participants in the present model understand perfectly their available actions (e.g. buy and sell orders) and the payoff relevant states (e.g. level of assets of a company). Moreover, they update using Bayes' rule and maximize their expected utility. However, they may be unaware of some contingencies which, although payoff irrelevant, could be correlated with contingencies that are payoff relevant. For example, a crisis in the housing market may influence the level of assets of several companies. Being unaware of a potential housing crisis means that the agent does not know whether it will occur and she does not know that she does not know. In other words, she completely misses this contingency and its effect on the payoff relevant states, viewing tomorrow's uncertainty in a simpler, more naive way.

To show that with unawareness the value of information may be positive for some, consider the previous example and suppose that additionally to the level of assets contingency, there is also the contingency of whether there is a housing market crisis. Moreover, suppose that the agents become aware of the second contingency if and only if the housing market crisis occurs and as a result the level of assets is low. At $a_{h}$, both agents do not know whether $a_{h}$ or $a_{l}$ has occurred because they have no information about the level of assets. Agent 1 agrees to give half of her endowment to agent 2 if $a_{h}$ is revealed, with the understanding that agent 2 will do the same if $a_{l}$ is revealed. As a result, both agents consume 1/2 at $a_{h}$. At $a_{l}$, both agents become aware of both contingencies. They can now reason that $a_{h}$ has not occurred, as it describes that they would be unaware of the housing crisis contingency, which is wrong. This implies that there is no trade and agent 2 consumes 1 , whereas agent 1 consumes 0 . If we compare this allocation with the full information allocation, where both consume their endowments, more information makes agent 1 strictly better off and agent 2 strictly worse off.

In Section 3 we provide numerical examples which demonstrate in more detail how more information can benefit some agents, at the expense of others. Moreover, we show an example with asymmetric awareness, where a more aware agent is strictly better off by revealing some of her awareness and information to the other agent, in order to manipulate her.

The common element in all these examples is that the value of information depends not on

\footnotetext{
${ }^{1}$ Schlee [2001] also identifies a third condition that we do not examine in this paper, namely that all agents are risk averse and the economy has a representative agent.

${ }^{2}$ See Andolfatto et al. [2014] and McTeer [2009] for a discussion.
} 
the level of awareness (how many contingencies one is aware of) but on its variance. If awareness varies across states, it creates an awareness signal that transmits information. However, the agent comprehends this signal only partially. For example, if she is aware of the housing market crisis contingency, she can easily exclude all states describing that she is unaware of it. On the other hand, if she is unaware of the housing market crisis contingency, then she cannot exclude the states which describe that she would be aware of it.

However, it is not necessary that there is perfect correlation between the level of awareness and the level of the assets, as in the previous example. We find that if there is some correlation between her level of awareness and the level of the assets which determines the endowment (e.g. she is more likely to become aware of the new contingency if the crisis occurs than if it does not), then her varying awareness provides information that is payoff relevant. The effect of the asymmetric use of the awareness signal is that risk-sharing opportunities that are mutually beneficial when the awareness signal is not used and information is scarce, vanish when the signal is used.

One way of avoiding this misuse of the awareness signal is to require that the agent's interim beliefs, which are the prior beliefs conditioned on her received information, do not change when we also condition on her awareness signal. We call this property Conditional Independence. Our main result is that Conditional Independence is sufficient for the value of public information to be negative for all fully insured risk averse agents, just like in the standard model. Moreover, we show that Conditional Independence is satisfied in the model of Heifetz et al. [2013], which is the probabilistic version of Heifetz et al. [2006]. Hence, the examples cited in Section 3 are excluded by these models.

Finally, although unawareness is a mistake of reasoning which leads agents to commit errors, it does not allow for any irrational behavior. For instance, we show that, as in the complete markets model of Green [1981], introducing public information to an uninformative information structure will lead to at least one agent being weakly worse off. This is a consequence of the First Welfare Theorem and it is still true with unawareness. This is not the case with other models that allow for arbitrary wrong beliefs (not necessarily due to unawareness), like Morris [1992] and Morris and Shin [1997].

\subsection{Related literature}

Using partitional structures, Green [1981] shows that if all agents are risk averse, when compared with no information, more information can never lead to an ex ante Pareto improvement. He also shows that the equilibrium outcomes of two information structures, one more informative than the other, cannot, in general, be ex ante Pareto ranked. Schlee [2001] proves that this is possible in the more restrictive environment of fully insured risk averse agents.

Campbell [2004] establishes that Hirshleifer's inverse relationship between better public information and welfare is fully general in the more general solution concept of implementable allocations. The value of information has also been studied in the setting of incomplete markets, where Green [1981], Hakansson et al. [1982] and Eckwert and Zilcha [2003] demonstrate that, in some cases, more information is beneficial. Gottardi and Rahi [2014] show that, generically, there exists a change in information that makes everyone better off and another change that makes everyone worse off.

Geanakoplos [1989] studies the value of information in a setting with boundedly rational agents, using non-partitional information structures on a single state space. Part of his motivation is unawareness, however Dekel et al. [1998] argue that unawareness cannot be modelled within a standard state space. Models of unawareness are provided, among others, by Fagin and Halpern [1988], Modica and Rustichini [1994, 1999], Halpern [2001], Li [2009], Halpern and Rêgo [2008], Heifetz et al. [2008] and Galanis [2011]. In this paper we use the multiple state space model of Galanis [2013], which is based on Heifetz et al. [2006]. Karni and Vierø [2013] 
provide a theory of how agents update their beliefs when their awareness grows and their state space expands.

In single-agent environments with unawareness, Galanis [2015] shows that the value of information may be negative, which is opposite to what is true when there is no unawareness, as shown in Blackwell [1951] and Laffont [1989]. He then identifies conditions on the awareness structure which are sufficient for the value to be positive. These conditions are weaker than the sufficient conditions in the current setting, for public information to have a negative value. In a similar setting, but where the value of information is always positive, Quiggin [2015] shows that the sum of the gains in expected utility, going from no information to some information and from minimal awareness to full awareness, is always constant. Li et al. [2014] examine the role of information disclosure in a model where a monopolist sells a product with a potentially harmful characteristic, of which some consumers are unaware.

The paper proceeds as follows. Section 2 presents the model and shows that Conditional Independence is sufficient for the value of public information to be negative for all agents. In Section 3 we provide examples, one with two risk averse agents and another where there is also a third risk neutral agent (e.g. insurer), which show that the value of public information may be positive for some agents and negative for others, if Conditional Independence is violated. We also show that an agent may become strictly better off by revealing some of her information and awareness to the other agent, in order to manipulate her. All proofs are contained in the Appendix.

\section{The Model}

\subsection{Preliminaries}

We present a reduced version of the model of Galanis [2013], which is based on Heifetz et al. [2006] and employs possibility correspondences $P^{i}$ but not knowledge or awareness operators. Differences in awareness are modeled by having several disjoint state spaces. Let $\mathcal{S}=\left\{S_{k}\right\}_{k \in K}$ be the collection of all these state spaces. For simplicity, we assume that $\mathcal{S}$ is finite and that each $S \in \mathcal{S}$ has finitely many elements, which we call states.

Figure 1 depicts an example, consisting of 4 state spaces. State space $S_{0}$ is the least expressive, containing only one state, denoted $\emptyset$. State space $S_{1}$ contains two states, describing whether the answer to question or dimension $p$ is yes or no, whereas $S_{2}$ describes whether the answer to $q$ is yes or no. State space $S_{3}$ is the most expressive, describing the answer to both $p$ and $q$. If an agent is aware of $S_{1}$ then she is also aware of $S_{0}$, but not necessarily of $S_{2}$ and $S_{3}$.

We assume that $\mathcal{S}$ is a lattice with partial order $\preceq$, so that for any two elements $S, S^{\prime} \in \mathcal{S}$, both their supremum $S \vee S^{\prime}$ and their infimum $S \wedge S^{\prime}$ belong to $\mathcal{S}$. In Figure 1, we have $S_{3}=S_{1} \vee S_{2}$ and $S_{0}=S_{1} \wedge S_{2}$. If $S \preceq S^{\prime}$, we say that $S^{\prime}$ is more expressive than $S$. Because $\mathcal{S}$ is finite, there is a top state space, which we call the full state space $S^{*}$, and a bottom state space, which we call the payoff relevant state space $S_{0}$. Hence, $S_{0} \preceq S \preceq S^{*}$, for all $S \in \mathcal{S}$. In the example, we have $S_{0} \prec S_{1}, S_{2}, S_{3}$ and $S_{3} \succ S_{0}, S_{1}, S_{2}$ but $S_{1}$ is not comparable with $S_{2}$, hence $S_{1} \nsucceq S_{2}$ and $S_{2} \nsucceq S_{1}$.

If $S \preceq S^{\prime}$, we require that each state $s^{\prime} \in S^{\prime}$ can be mapped to its "restricted" image in the less expressive $S$. Formally, we require that there is a surjective projection $r_{S}^{S^{\prime}}: S^{\prime} \rightarrow S$. Projections are required to commute: if $S \preceq S^{\prime} \preceq S^{\prime \prime}$, then $r_{S}^{S^{\prime \prime}}=r_{S}^{S^{\prime}} \circ r_{S^{\prime}}^{S^{\prime \prime}}$. We denote the projection of set $E^{\prime} \subseteq S^{\prime}$ to the less expressive $S$ by $E_{S}^{\prime}=\bigcup\left\{r_{S}^{S^{\prime}}\left(s^{\prime}\right) \in S: s^{\prime} \in E^{\prime}\right\}$. We denote the enlargement of $E^{\prime} \subseteq S^{\prime}$ to the more expressive $S^{\prime \prime}$ by $E^{\prime S^{\prime \prime}}=\bigcup\left\{s^{\prime \prime} \in S^{\prime \prime}: r_{S^{\prime}}^{S^{\prime \prime}}\left(s^{\prime \prime}\right) \in E^{\prime}\right\}$. Let $E^{\uparrow}=\bigcup\left\{E^{\prime S^{\prime \prime}}: S^{\prime \prime} \succeq S^{\prime}\right\}$ be the enlargements of $E^{\prime}$ to all state spaces which are at least as expressive as $S^{\prime}$.

In Figure 1, the thin arrows show the projections. State $p_{y} q_{y}$ of $S_{3}$ projects to state $p_{y}$ of 


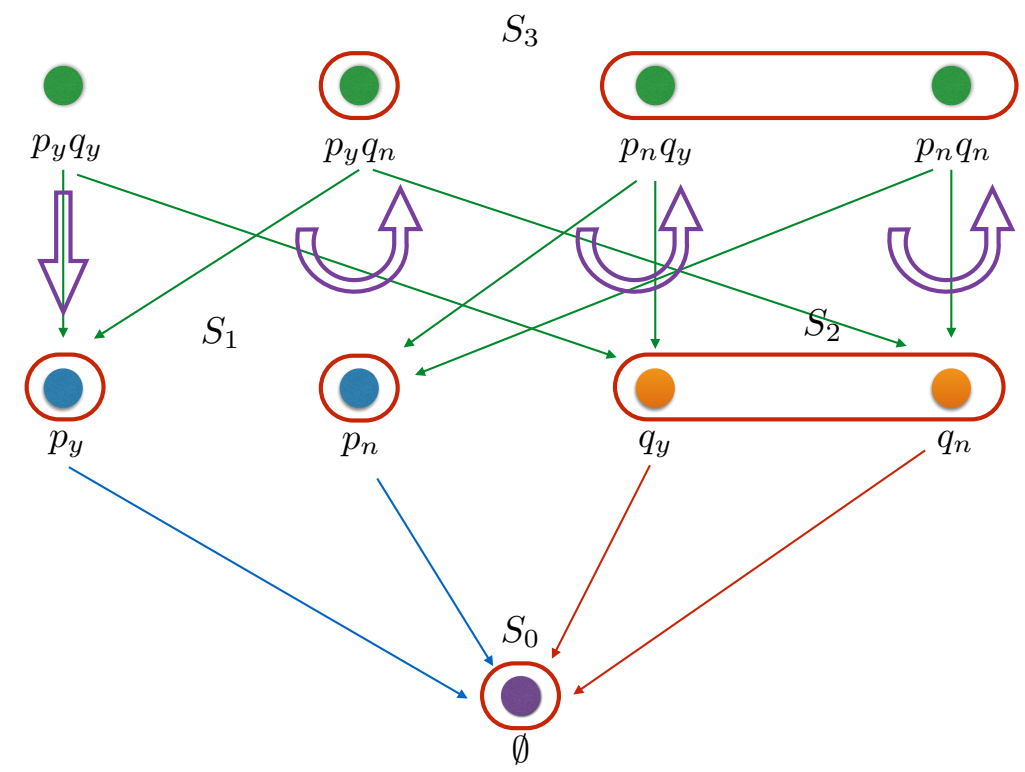

Figure 1: Collection of state spaces

$S_{1}$ and to state $q_{y}$ of $S_{2}$. States $p_{y}$ and $q_{y}$ then project to state $\emptyset$ of $S_{0}$. The projection of $E=\left\{p_{y} q_{y}, p_{n} q_{y}\right\}$ to $S_{1}$ is $E_{S_{1}}=\left\{p_{y}, p_{n}\right\}=S_{1}$. However, the enlargement of $E_{S_{1}}$ to $S_{3}$ is $\left(E_{S_{1}}\right)^{S_{3}}=S_{3}$.

\subsection{Information and awareness}

Agent $i$ 's information structure is represented by a possibility correspondence $P^{i}: \Sigma \rightarrow 2^{\Sigma} \backslash \emptyset$, where $\Sigma=\bigcup_{S \in \mathcal{S}} S$ is the set of all states. The interpretation is that $i$ considers states in $P^{i}(s)$ to be possible at $s \in S$. We assume that $P^{i}$ has the following properties.

(0) Confinedness: If $s \in S$ then $P^{i}(s) \subseteq S^{\prime}$ for some $S^{\prime} \preceq S$.

(1) Generalized Reflexivity: $s \in\left(P^{i}(s)\right)^{\uparrow}$ for every $s \in \Sigma$.

(2) Stationarity: $s^{\prime} \in P^{i}(s)$ implies $P^{i}\left(s^{\prime}\right)=P^{i}(s)$.

(3) Projections Preserve Ignorance: If $s \in S^{\prime}$ and $S \preceq S^{\prime}$ then $\left(P^{i}(s)\right)^{\uparrow} \subseteq\left(P^{i}\left(s_{S}\right)\right)^{\uparrow}$.

(4) Projections Preserve Awareness: If $s \in S^{\prime}, s \in P^{i}(s)$ and $S \preceq S^{\prime}$ then $s_{S} \in P^{i}\left(s_{S}\right)$.

Detailed discussion of these properties is provided in Heifetz et al. [2006] and Galanis [2013]. In Figure 1, the thick arrows specify what is the agent's awareness and state space at each full state. For example, state $p_{y} q_{y}$ specifies that the agent is aware of $p$ but not $q$, hence her state space is $S_{1}$. All other states in $S^{*}$ specify that she is aware of both $p$ and $q$ and her state space is $S^{*}$. The solid lines specify the agent's information at each state. In particular, we assume that the agent receives a perfectly correlated signal about $p$, meaning that if she is aware of $p$, she is informed whether the answer is yes or no. For instance, at $p_{n} q_{y}$ she is aware of state space $S_{3}$ (and therefore all less expressive state spaces) and she considers $p_{n} q_{y}$ and $p_{n} q_{n}$ to be possible because she learns the answer to $p$ is no. Hence, $P^{i}\left(p_{n} q_{y}\right)=\left\{p_{n} q_{y}, p_{n} q_{n}\right\}$. The thick arrow pointing from $p_{y} q_{y}$ to $p_{y}$ indicates that if $p_{y} q_{y}$ occurs, then the agent's state space is $S_{1}$, hence she is unaware of $S_{3}$ and $S_{2}$. Because she is informed about $p$, she knows that $p_{y}$ is true and $P^{i}\left(p_{y} q_{y}\right)=\{p\}$. State $p_{y} q_{y}$ is not included in any solid line because the agent never considers 
it possible. When it occurs, she is unaware of it. When she is aware of it (by being aware of $S_{3}$ ), she understands that $p_{y} q_{y}$ could not have happened, because it specifies that her awareness would be lower.

In the standard model without unawareness, we say that information structure $P^{1}$ is more informative than $P^{2}$ if for all states $s \in S$ (where $S$ is the unique state space), $P^{1}(s) \subseteq P^{2}(s)$. This property is equivalent to saying that the partition generated by $P^{1}$ is finer than that generated by $P^{2}$. In a model with unawareness, however, it may be that $P^{1}(s)$ and $P^{2}(s)$ do not belong to the same state space. In order to compare them, we enlarge both sets to a state space $S$ that is more expressive than both $P^{1}(s)$ and $P^{2}(s)$, for all $s \in S$.

Definition 1. $P^{2}$ is more informative than $P^{1}$ given $S \in \mathcal{S}$ if $P^{2}(s)^{S} \subseteq P^{1}(s)^{S}$ for all $s \in S .^{3}$

Hence, the definition of "more information" is parametrized by state space $S$. Suppose that $P^{1}$ is the information structure depicted in Figure 1 and define $P^{2}$ as follows. For each $s \in S_{3}$, $P^{2}(s)=s_{S_{1}}$, hence $S_{3}$ specifies that agent 2 is always aware of $S_{1}$ and she is perfectly informed about what the state is. For example, $P^{2}\left(p_{y} q_{y}\right)=\left\{p_{y}\right\}$. For each $s \in S_{0} \cup S_{1} \cup S_{2}, P^{2}(s)=\{s\}$. Then, $P^{1}$ is more informative than $P^{2}$ given $S_{3}$ but $P^{2}$ is not more informative than $P^{1}$ given $S_{3}$, because $P^{1}\left(p_{y} q_{n}\right)^{S_{3}} \subsetneq P^{2}\left(p_{y} q_{n}\right)^{S_{3}}$. Given $S_{1}$, however, $P^{1}$ is identical to $P^{2}$.

An event $E$ is a subset of some state space $S \in \mathcal{S}$. Hence, $\left\{p_{y} q_{y}, p_{y} q_{n}\right\}$ is an event but $\left\{p_{y}, p_{n} q_{y}\right\}$ is not. Define $S^{i}: \Sigma \rightarrow \mathcal{S}$ such that for any $s \in \Sigma, S^{i}(s)=S$ if $P^{i}(s) \subseteq S \in \mathcal{S}$. Hence, $S^{i}(s)$ is the most expressive state space that the agent is aware of at $s$ and we therefore say that $S^{i}(s)$ is $i$ 's state space at $s$.

Definition 2. Agent $i$ is aware of event $E \subseteq S^{\prime}$ at $s \in S$ if $S^{\prime} \preceq S^{i}(s)$. If $S^{i}(s) \succeq S^{j}(s)$, then agent $i$ is more aware than agent $j$ at $s$.

In Figure $1, S^{1}\left(p_{y} q_{y}\right)=S_{1}$ so she is aware of event $\left\{p_{y}\right\}$ but not $\left\{p_{y} q_{y}, p_{y} q_{n}\right\}$. She is aware of all events at $p_{y} q_{n}$ because $S^{1}\left(p_{y} q_{n}\right)=S_{3}$. Moreover, agent 1 is at least as aware as agent 2 at all states $s \in \Sigma$.

\subsection{Priors and updating}

Let $\pi_{0}$ be a prior on the full state space $S^{*}$. We assume that if the agent's state space is $S \preceq S^{*}$, her prior is just the marginal of $\pi_{0}$ on $S$. Hence, being less aware does not imply a different prior. In Figure 1, let $\pi_{0}=(0.2,0.2,0.4,0.2)$ be the prior on $S^{*}=S_{3}$. At $p_{y} q_{y}$, the agent's state space is $S_{1}$, as shown by the arrow. Her prior on $S_{1}$ is the marginal of $\pi_{0}$ on $S_{1}$, which is $(0.4,0.6)$. Formally, for each $S \in \mathcal{S}$, define $\pi_{S}: 2^{S} \backslash \emptyset \rightarrow[0,1]$, such that, for each event $E \subseteq S$, $\pi_{S}(E)=\pi_{0}\left(E^{S^{*}}\right)$. We call $\pi=\left\{\pi_{S}\right\}_{S \in \mathcal{S}}$ a generalized prior.

Posterior beliefs are formulated using Bayes' rule. If $i$ is aware of event $E$ at state $s$ and $\pi_{S}\left(P^{i}(s)\right)>0$, where $S=S^{i}(s)$, then her posterior belief about $E \preceq S$ at $s$ is $\pi_{S}\left(E^{S} \mid P^{i}(s)\right)=$ $\frac{\pi_{S}\left(E^{S} \cap P^{i}(s)\right)}{\pi_{S}\left(P^{i}(s)\right)}$. In Figure 1, the agent's information at $p_{n} q_{y}$ is $P^{i}\left(p_{n} q_{y}\right)=\left\{p_{n} q_{y}, p_{n} q_{n}\right\}$. Her posterior belief about $E=\left\{q_{y}\right\}$ at $p_{n} q_{y}$ is $\pi_{S_{3}}\left(E^{S_{3}} \mid P^{i}\left(p_{n} q_{y}\right)\right)=\frac{\pi_{S_{3}}\left(\left\{p_{n} q_{y}\right\}\right)}{\pi_{S_{3}}\left(\left\{p_{n} q_{y}, p_{n} q_{n}\right\}\right)}=0.67$.

\subsection{Conditional Independence}

Given state $s \in S \in \mathcal{S}$, let

$$
\mathcal{E}_{S}^{i}(s)=\left\{s_{1} \in S: S^{i}(s)=S^{i}\left(s_{1}\right)\right\}
$$

be the event in $S$ describing that the agent has the same awareness as in $s$. Operator $\mathcal{E}_{S}^{i}$ partitions state space $S$, generating an awareness signal, expressed in $S$, that provides information. We call $\mathcal{E}^{i}=\left\{\mathcal{E}_{S}^{i}\right\}_{S \in \mathcal{S}}$ the awareness signal of $P^{i}$. At $s$, the agent's state space is $S^{i}(s)$. Properties 0-4 of Section 2.2 imply that the agent always knows what her awareness is. This means that she

\footnotetext{
${ }^{3}$ To ease the notation we write $P^{1}(s)^{S}$ instead of $\left(P^{1}(s)\right)^{S}$.
} 
considers impossible any state in $S^{i}(s)$ that describes that her awareness would be strictly lower than $S^{i}(s)$. These states are precisely those not in $\mathcal{E}_{S^{i}(s)}^{i}\left(s_{S^{i}(s)}\right)$, where $s_{S^{i}(s)}$ is the projection of $s$ to her state space. In other words, it is as if the agent is informed that $\mathcal{E}_{S^{i}(s)}^{i}\left(s_{S^{i}(s)}\right)$ is true and incorporates it in her information, hence $P^{i}(s) \subseteq \mathcal{E}_{S^{i}(s)}^{i}\left(s_{S^{i}(s)}\right)$ is always true. However, if $S \succeq S^{\prime} \succ S^{i}(s)$, then $\mathcal{E}_{S^{\prime}}^{i}\left(s_{S^{\prime}}\right)$ as a signal is more informative and the agent is unaware of it.

In Figure $1, \mathcal{E}_{S_{3}}^{1}\left(p_{y} q_{y}\right)=\left\{p_{y} q_{y}\right\}$, whereas if $s \in S_{3}$ is different from $p_{y} q_{y}$ we have $\mathcal{E}_{S_{3}}^{1}(s)=$ $\left\{p_{n} q_{y}, p_{y} q_{n}, p_{n} q_{n}\right\}$. For all states $s \in S_{3}$ that are different from $p_{y} q_{y}$, the agent is aware of $S_{3}$. As a result, she completely understands $\mathcal{E}_{S_{3}}^{1}$, which is already incorporated in her information, in the sense that $P^{1}(s) \subseteq \mathcal{E}_{S_{3}}^{1}(s)$ for all $s \in S \backslash p_{y} q_{y}$. At $p_{y} q_{y}$, however, she is unaware of $S_{3}$, which means that she cannot understand $\mathcal{E}_{S_{3}}^{1}$. This means that she cannot reason as follows: "because I am unaware of $S_{3}$, it must be that $p_{y} q_{y}$ has occurred". This reasoning, expressed in state space $S_{1}$, implies that $p_{y}$ has occurred. Does the agent lose in terms of her information by not being able to reason above her awareness? In this particular case no, because she already knows that $p_{y}$ has occurred as she receives a signal, but in general this is not true, as we show in Section 3.

We formalise this property below but express it in terms of beliefs, which matter for actions, instead of information. Conditional Independence given state space $S$ specifies that interim beliefs would be the same even if the agent could use her awareness signal, expressed in $S$. In particular, it specifies that interim beliefs at $s \in S$, which are the prior beliefs conditioned on $P^{i}(s)$, do not change when we also condition on $\mathcal{E}_{S}^{i}(s)$, the awareness signal of $P^{i}$, expressed in $S$.

Definition 3. $\left(P^{i}, \pi\right)$ satisfies Conditional Independence given $S \in \mathcal{S}$ if, for any $s \in S$ with $\pi_{S}(s)>0$, for any $E \subseteq S^{i}(s)$,

$$
\pi_{S^{i}(s)}\left(E \mid P^{i}(s)\right)=\pi_{S}\left(E^{S} \mid \mathcal{E}_{S}^{i}(s) \cap P^{i}(s)^{S}\right) .
$$

In Figure 1, let $E=\left\{p_{y}\right\}$ and note that the agent's interim beliefs about $E$ at $p_{y} q_{y} \in S_{3}$ are $\pi_{S^{1}}\left(E \mid P^{1}\left(p_{y} q_{y}\right)\right)=1$, because she knows whether $p$ is true or not. The awareness signal of $P^{1}$, expressed in $S_{3}$, specifies that $\mathcal{E}_{S_{3}}^{1}\left(p_{y} q_{y}\right)=\left\{p_{y} q_{y}\right\}$, hence $\pi_{S_{3}}\left(E^{S_{3}} \mid \mathcal{E}_{S_{3}}^{1}\left(p_{y} q_{y}\right) \cap P^{1}\left(p_{y} q_{y}\right)^{S_{3}}\right)=$ 1. For all other $s \in S_{3} \backslash p_{y} q_{y}$, we have $\mathcal{E}_{S_{3}}^{1}(s) \supseteq P^{1}(s)$, hence $\left(P^{1}, \pi\right)$ satisfies Conditional Independence given $S_{3}$. In Section 3, we provide examples where Conditional Independence is violated and, as a result, the value of public information is positive for some agents.

As we show below, Conditional Independence is sufficient for public information to have negative value for all, as in the standard model. To provide a connection with the unawareness literature, consider the following property of Heifetz et al. [2013] (Section 2.8, Property 2), which extends the unawareness model of Heifetz et al. [2006] by introducing probabilistic beliefs. Translated in the current setting, it is the following. ${ }^{4}$

Definition 4. $\left(P^{i}, \pi\right)$ satisfies Projections Preserve Posteriors if, for all $s \in \Sigma$, if $S^{\prime \prime} \succeq S^{\prime} \succeq S$, $s \in S^{\prime \prime}$ and $S^{i}(s)=S^{\prime}$, then for any event $E \subseteq S, \pi_{S}\left(E \mid P^{i}\left(s_{S}\right)\right)=\pi_{S^{\prime}}\left(E^{S^{\prime}} \mid P^{i}(s)\right) .^{5}$

Projections Preserve Posteriors requires that if both $s$ and its projection to $S, s_{S}$, describe that the agent is aware of event $E$, then both $s$ and $s_{S}$ specify the same posterior beliefs about $E$. This property is a strengthening of the property Projections Preserve Knowledge, assumed in Heifetz et al. [2006] but not in Galanis [2013]. The next proposition shows that it is stronger than Conditional Independence.

Proposition 1. If $\left(P^{i}, \pi\right)$ satisfies Projections Preserve Posteriors, then it satisfies Conditional Independence given $S$, for each $S \in \mathcal{S}$.

\footnotetext{
${ }^{4}$ The name Projections Preserve Posteriors is not used by Heifetz et al. [2013].

${ }^{5}$ Note that Projections Preserve Awareness implies that $S^{i}\left(s_{S}\right)=S$.
} 
In conjunction with Theorem 1, stated in Section 2.6, Proposition 1 says that the value of public information is always negative for all agents in the model of Heifetz et al. [2013].

Finally, note that Conditional Independence does not imply Projections Preserve Posteriors. For a counterexample, suppose $\mathcal{S}=\left\{S, S^{*}\right\}$, each state space containing two states, where $s_{k}^{*} \in S^{*}$ projects to $s_{k} \in S, i=1,2$. Moreover, $P^{i}\left(s_{k}^{*}\right)=\left\{s_{k}^{*}\right\}, P^{i}\left(s_{k}\right)=S, i=1,2 .{ }^{6}$ With a uniform prior on $S^{*}$, Conditional Independence given both $S^{*}$ and $S$ is satisfied, because the agent's awareness is constant. However, Projections Preserve Posteriors is violated because at $s_{1}$ she assigns probability $1 / 2$ to event $E=\left\{s_{1}\right\}$, but at $s_{1}^{*}$ she assigns probability 1 to $E^{S^{*}}$.

\subsection{The economy}

Consider an exchange economy with $I$ agents and a single physical consumption good. Endowments are defined on the bottom, payoff relevant, state space $S_{0}$. Agent $i$ 's endowment at $s \in S_{0}$ is $\omega^{i}(s)$. The aggregate endowment of the economy at state $s \in S_{0}$ is $\sum_{i \in I} \omega^{i}(s)=\omega(s)$.

For any other state space $S \in \mathcal{S}$, $i$ 's endowment at $s \in S$ is $\omega^{i}(s)=\omega^{i}\left(s_{S_{0}}\right)$, where $s_{S_{0}} \in S_{0}$ is the projection of $s$ to state space $S_{0}$. Hence, all other state spaces are payoff irrelevant. There is no aggregate uncertainty if $\omega(s)=\omega\left(s^{\prime}\right)$ for all $s, s^{\prime} \in S_{0}$, so that the aggregate endowment is constant across all states in $S_{0}$. Let $\omega^{i}=\left\{\omega^{i}(s)\right\}_{s \in \Sigma}$ be the vector specifying the endowments for agent $i$ in all possible states. Let $\left\{\omega^{i}\right\}_{i \in I}$ be the vector specifying the endowments for all agents. Each agent has a differentiable, concave, strictly increasing von Neumann-Morgestern utility function $u^{i}: \mathbb{R}_{+} \rightarrow \mathbb{R}$. Let $\left\{u^{i}\right\}_{i \in I}$ be the vector of utilities for all agents.

There are three periods, 0 and 1 and 2. Period 0 is the ex ante period, before receiving any signal. In period 1, the agents receive their awareness and information, update using Bayes' rule and trade conditioning on the payoff relevant states $s_{0} \in S_{0}$. In period 2 , the true payoff relevant state is revealed, enabling the execution of the agreed trades.

Information and awareness are common among agents, so that $P^{i}=P^{j}=P$ for all $i, j \in I$. An economy is denoted by a tuple $\left(S, P,\left\{\omega^{i}\right\}_{i \in I},\left\{u^{i}\right\}_{i \in I}, \pi\right)$, where $P$ describes the agents' (common) information and awareness, $\left\{\omega^{i}\right\}_{i \in I}$ is the endowment vector, $\left\{u^{i}\right\}_{i \in I}$ is the utility vector, $\pi$ is the common generalized prior and $S \in \mathcal{S}$ is the state space that is used to describe the uncertainty in period 1. That is, an agent whose state space is $S$ in period 0 thinks that in period 1 the economy is described by tuple $\left(S, P,\left\{\omega^{i}\right\}_{i \in I},\left\{u^{i}\right\}_{i \in I}, \pi\right)$. When $s \in S$ occurs, the agents' state space is $S(s)$ and the public information is given by $P(s)$. For simplicity, we assume that $\pi_{S}(s)>0$ for all $s \in S \in \mathcal{S}$.

Given a state $s \in S \in \mathcal{S}$ and a possibility correspondence $P$, let $c_{s}^{i}\left(s_{1}\right) \in \mathbb{R}_{+}$denote agent $i$ 's consumption at $s_{1} \in P(s)$, if the public information is $P(s) .{ }^{7}$ Let $c_{s}^{i}=\left\{c_{s}^{i}\left(s_{1}\right)\right\}_{s_{1} \in P(s)}$ be a vector of consumptions for agent $i$ for all states considered possible given $s \in S$. Vector $\left\{c_{s}^{i}\right\}_{i \in I}$ is feasible if, for each $s_{1} \in P(s), \sum_{i \in I} c_{s}^{i}\left(s_{1}\right) \leq \sum_{i \in I} \omega^{i}\left(s_{1}\right)$, where $\omega^{i}\left(s_{1}\right)=\omega^{i}\left(s_{1 S_{0}}\right)$ is $i$ 's endowment at $s_{1}$.

Let $c_{S, P}^{i}=\left\{c_{s}^{i}\right\}_{s \in S}$ summarize $i$ 's consumption for all states in $S$ given $P$ and let $c_{S, P}=$ $\left\{c_{S, P}^{i}\right\}_{i \in I}$ summarize the consumption of all agents. Vector $c_{S, P}$ is feasible if $\left\{c_{s}^{i}\right\}_{i \in I}$ is feasible for each $s \in S$. It is measurable if the following two conditions hold for all $i \in I$. First, for

\footnotetext{
${ }^{6}$ Although it is not necessary to describe specific dimensions for the two state spaces, suppose that $s_{1}=\left\{p_{y}\right\}$, $s_{2}=\left\{p_{n}\right\}, s_{1}^{*}=\left\{p_{y} q_{y}\right\}$ and $s_{2}^{*}=\left\{p_{n} q_{n}\right\}$. The information structure can then be derived by specifying that at $s_{k}^{*} \in S^{*}$, the agent learns the answer to $q$ and uses the theorem " $q_{y}$ if and only $p_{y}$ " to deduce the answer to $p$ and learn the true state. Because state space $S$ does not contain dimension $q$ and therefore misses this connection, or theorem, it describes that the agent has no information about $p$. Note that to express this theorem, the full state space is not the Cartesian product of the answers of all possible questions. Galanis [2013] studies these theorems and their impact on interactive knowledge, in an environment with unawareness.

${ }^{7}$ Note that we do not define consumption for states which do not belong to $P(s)$, because they are considered impossible by all agents, when the public information is $P(s)$.
} 
all $s \in S$, for all $s_{1}, s_{2} \in P(s)$ such that $s_{1 S_{0}}=s_{2 S_{0}}$, we have $c_{s}^{i}\left(s_{1}\right)=c_{s}^{i}\left(s_{2}\right)$. Second, for all $s, s^{\prime} \in S$ such that $P(s)=P\left(s^{\prime}\right)$, we have $c_{s}^{i}\left(s_{1}\right)=c_{s^{\prime}}^{i}\left(s_{1}\right)$ for all $s_{1} \in P(s)=P\left(s^{\prime}\right)$. In other words, $c_{S, P}$ is measurable if it is measurable with respect to the payoff relevant state space $S_{0}$ and with respect to any two signals that are identical.

Given public information $P(s)$, the price of the state-contingent good at state $s_{1} \in P(s)$ is denoted by $p_{s}\left(s_{1}\right)$. Let $p_{s}=\left\{p_{s}\left(s_{1}\right)\right\}_{s_{1} \in P(s)}$ be a vector of prices for all states considered possible at $s$ and let $p_{S, P}=\left\{p_{s}\right\}_{s \in S}$ summarize prices for all states in $S$ given $P$. Note that, unlike in the standard model, allocation $c_{S, P}$ and prices $p_{S, P}$ are defined with reference to both a state space $S$ and a possibility correspondence $P$. The reason is that $P$ determines the agents' awareness and therefore their state space $S(s)$, when $s \in S$ occurs. Consumption and prices are then defined for all states in $P(s)$. Vector $p_{S, P}$ is measurable if for all $s, s^{\prime} \in S$, if $P(s)=P\left(s^{\prime}\right)$ then $p_{s}=p_{s^{\prime}}$.

Vector $c_{s}^{i}$ is affordable for $i$ given price vector $p_{s}$ if $\sum_{s_{1} \in P(s)} c_{s}^{i}\left(s_{1}\right) p_{s}\left(s_{1}\right) \leq \sum_{s_{1} \in P(s)} \omega^{i}\left(s_{1}\right) p_{s}\left(s_{1}\right)$.

We say that $\left\{c_{s}^{i}\right\}_{i \in I}$ is affordable given $p_{s}$ if, for each $i \in I, c_{s}^{i}$ is affordable for $i$ given $p_{s}$.

Given state space $S$ and information structure $P$, we evaluate allocation $c_{S, P}^{i}$ from an ex ante perspective. If state $s \in S$ occurs, the agents' state space is $S(s)$ and $i$ 's consumption under $c_{S, P}^{i}$ is $c_{s}^{i}\left(s_{S(s)}\right)$. Her ex ante expected utility is $\sum_{s \in S} \pi_{S}(s) u^{i}\left(c_{s}^{i}\left(s_{S(s)}\right)\right)$.

A competitive equilibrium in a risk-sharing environment with symmetric awareness and information is defined as follows.

Definition 5. Given an economy $\left(S, P,\left\{\omega^{i}\right\}_{i \in I},\left\{u^{i}\right\}_{i \in I}, \pi\right)$, a competitive equilibrium consists of an allocation $c_{S, P}$ and a price vector $p_{S, P}$ such that:

- Agent optimization: For all $i \in I$, for all $s \in S$, for any vector of consumptions $\left\{d_{s}^{i}\right\}_{i \in I}$ which is affordable given $p_{s}$ and measurable, we have

$$
\sum_{s_{1} \in P(s)} \pi_{S(s)}\left(s_{1} \mid P(s)\right) u^{i}\left(c_{s}^{i}\left(s_{1}\right)\right) \geq \sum_{s_{1} \in P(s)} \pi_{S(s)}\left(s_{1} \mid P(s)\right) u^{i}\left(d_{s}^{i}\left(s_{1}\right)\right) .
$$

- Measurability: $c_{S, P}$ and $p_{S, P}$ are measurable.

- Market clearing: For each $s \in S$, for each $s_{1} \in P(s), \sum_{i \in I} c_{s}^{i}\left(s_{1}\right)=\sum_{i \in I} \omega^{i}\left(s_{1}\right)$.

Note that a competitive equilibrium is defined given a state space $S$. Moreover, given a state $s \in S$, the agents have received their information and awareness before making their decisions about their trades. Hence, given $s$ we are in a standard case of an equilibrium under uncertainty, where the state space is $P(s)$. The added condition of measurability comes from the fact that we represent information using possibility correspondences, not signals. Hence, unawareness does not add any complications to the notion of equilibrium.

\subsection{Main result}

Before providing sufficient conditions for information not to be valuable in a risk-sharing environment, we confirm a result by Green [1981], showing that from an initial position of no information, any public information will result in at least one agent being weakly worse off ex ante. This is a consequence of the First Welfare Theorem. Moreover, if that agent is risk averse and her consumption is not constant conditional on each payoff relevant state $s_{0} \in S_{0}$, then she will be strictly worse off ex ante. This result shows that unawareness does not imply that "anything goes", as in the case where agents are irrational in an arbitrary way. ${ }^{8}$

\footnotetext{
${ }^{8}$ For example, Morris [1992] and Morris and Shin [1997] allow agents to misunderstand their signals in an arbitrary (and unmodelled) way, hence being less restrictive in terms of behavior, when compared to the present model. As a result, Proposition 2 is not true in their model.
} 
We say that $P$ is uninformative given state space $S$ if $P(s)=P\left(s^{\prime}\right)$, for all $s, s^{\prime} \in S$. This condition implies that awareness is constant for all states in $S$, hence the awareness signal is uninformative as well. Any possibility correspondence $P^{\prime}$ is more informative than an uninformative $P$.

Recall that in economy $\left(S, P,\left\{\omega^{i}\right\}_{i \in I},\left\{u^{i}\right\}_{i \in I}, \pi\right)$ all agents have the same information, given by possibility correspondence $P$. We compare how agents evaluate ex ante their consumption from the equilibrium allocation in economies $\left(S, P^{1},\left\{\omega^{i}\right\}_{i \in I},\left\{u^{i}\right\}_{i \in I}, \pi\right)$, where $P^{1}$ is uninformative given $S$, and $\left(S, P^{2},\left\{\omega^{i}\right\}_{i \in I},\left\{u^{i}\right\}_{i \in I}, \pi\right)$. We show that at least one agent will be weakly worse off in economy $\left(S, P^{2},\left\{\omega^{i}\right\}_{i \in I},\left\{u^{i}\right\}_{i \in I}, \pi\right)$ and, if she is strictly risk averse and her consumption is not constant across all states, strictly worse off.

Proposition 2. Consider a competitive equilibrium allocation $c_{S, P^{1}}$ of economy $\left(S, P^{1},\left\{\omega^{i}\right\}_{i \in I},\left\{u^{i}\right\}_{i \in I}, \pi\right)$, where $P^{1}$ is uninformative given $S$, and a competitive equilibrium allocation $d_{S, P^{2}}$ of economy $\left(S, P^{2},\left\{\omega^{i}\right\}_{i \in I},\left\{u^{i}\right\}_{i \in I}, \pi\right)$. Then, for some $i \in I$, $i$ 's attained level of ex ante expected utility must be weakly lower under $d_{S, P^{2}}$ than under $c_{S, P^{1}}$. Moreover, if $u^{i}$ is strictly concave and $i$ 's consumption under $d_{S, P^{2}}$ is not constant conditional on each payoff relevant state, then $i$ 's attained level of ex ante expected utility must be strictly lower.

As Green [1981] points out, we cannot generalize this proposition by only requiring that $P^{2}$ is more informative than (a not necessarily uninformative) $P^{1}$. The reason is that, although each cell of $P^{1}$ is partitioned by cells of the finer $P^{2}$, allowing us to apply the proposition on each cell separately, the weakly worse off agent may not be the same across all cells. Hence, taking the expectation with respect to the whole state space may not identify an agent who is weakly worse off.

Schlee [2001] shows that more public information will make everyone weakly worse off in the more restrictive environment of fully insured risk averse agents, which is implied by two separate conditions. ${ }^{9}$ First, there is no aggregate uncertainty. Second, there are risk neutral agents, rich enough to fully insure the risk averse agents. ${ }^{10}$

As is shown with examples in Section 3, both these conditions are no longer sufficient with unawareness. The following theorem shows that we need to additionally assume Conditional Independence for both information structures.

Theorem 1. Suppose that $P^{2}$ is more informative than $P^{1}$. Moreover, both $\left(P^{1}, \pi\right)$ and $\left(P^{2}, \pi\right)$ satisfy Conditional Independence given $S$. Consider a competitive equilibrium allocation $c_{S, P^{1}}$ of economy $\left(S, P^{1},\left\{\omega^{i}\right\}_{i \in I},\left\{u^{i}\right\}_{i \in I}, \pi\right)$ and a competitive equilibrium allocation $d_{S, P^{2}}$ of economy $\left(S, P^{2},\left\{\omega^{i}\right\}_{i \in I},\left\{u^{i}\right\}_{i \in I}, \pi\right)$. Then, all agents are weakly worse off under $d_{S, P^{2}}$ if either of the following is true:

1. All agents are risk averse and there is no aggregate uncertainty, or,

2. There are enough risk neutral agents who can fully insure the risk averse agents.

Conditions 1 and 2 ensure that the risk averse agents are fully insured in all equilibrium allocations. It is a standard result that full insurance and risk aversion imply that there is a unique equilibrium allocation, specifying that at the interim stage each risk averse agent consumes her expected endowment. ${ }^{11}$ Conditional Independence on both information structures then implies that more information will make everyone weakly worse off. The two counterexamples of Section 3 show that we need both $P^{1}$ and $P^{2}$ to satisfy Conditional Independence for the value of information to be negative for all.

\footnotetext{
${ }^{9}$ Note that Schlee [2001] uses Blackwell's criterion of "more information", which is different than the one used here and in Green [1981]. The relation between the two is shown in Green and Stokey [1978].

${ }^{10}$ As stated before, Schlee [2001] also identifies a third condition that we do not examine here, namely that all agents are risk averse and the economy has a representative agent.

11 See Theorem 2 in Schlee [2001]. We provide a sketch of the proof of this result in the next section.
} 


\section{Positive value of public information}

We now provide two counterexamples, showing that if Conditional Independence is violated, then the conditions of Schlee [2001] are no longer sufficient for public information to have negative value in a risk-sharing environment with unawareness, where a single good is traded. In the first example there are two risk averse agents and no aggregate uncertainty, whereas in the second there is aggregate uncertainty and a third, risk neutral, agent who fully insures the first two. In both cases, we also show that if awareness and information are asymmetric, then it is possible for an agent to intentionally make some of her awareness and information public, in order to manipulate the other agents.

Uncertainty about tomorrow is described by giving an answer to the following three questions, which concern a specific company. Question a, "What is the level of the assets of the company?", has three possible answers: high, medium and low. Questions $q$, "Is there an acquisition?", and $c$, "Is there a housing market crisis?", have two possible answers, "yes" and "no".

A full state $s^{*}$ specifies an answer to all three questions, providing a complete description of the world. The full state space $S^{*}=\left\{s_{1}^{*}, s_{2}^{*}, s_{3}^{*}\right\}$ consists of states

$$
s_{1}^{*}=\left(a_{h}, q_{y}, c_{n}\right), s_{2}^{*}=\left(a_{m}, q_{n}, c_{n}\right) \text { and } s_{3}^{*}=\left(a_{l}, q_{y}, c_{y}\right) .
$$

The full state space describes that the level of assets is low if and only if there is a housing market crisis. Moreover, the level of assets is medium if and only if there is no acquisition. The first connection is lost for an agent who is only aware of questions $a$ and $q$. Her state space is then $S=\left\{s_{1}, s_{2}, s_{3}\right\}$, the projection of $S^{*}$ to this lower dimensional space. It consists of states

$$
s_{1}=\left(a_{h}, q_{y}\right), s_{2}=\left(a_{m}, q_{n}\right) \text { and } s_{3}=\left(a_{l}, q_{y}\right) .
$$

Finally, the payoff relevant state space $S_{0}=\left\{a_{h}, a_{m}, a_{l}\right\}$ specifies an answer only to question $a$. The common prior on each of the three state spaces is given by $(0.3,0.3,0.4)$.

At each full state $s^{*} \in S^{*}$, the agents receive their awareness and information, which is common, before trading. Hence, they have a common state space and common posteriors. In this interim stage, there is no difference with a standard general equilibrium model under uncertainty, where agents trade a state-contingent good after receiving a public signal. In both subsequent examples, the risk averse agents are fully insured, which implies that the unique equilibrium allocation is to consume their expected endowment, given their common interim beliefs.

To see why this is the case, suppose that after receiving their common awareness and information, the agents consider all states in $S$ to be possible. Suppose that all agents are risk averse and there is no aggregate uncertainty. Each agent $i$ maximises $\sum_{s \in S} \pi_{S}(s) u^{i}\left(c^{i}(s)\right)$, subject to $\sum_{s \in S} p(s) c^{i}(s)=\sum_{s \in S} p(s) \omega^{i}(s)$. No aggregate uncertainty implies that $\sum_{i \in I} \omega^{i}(s)=\omega$ for each $s \in S$. Consider a competitive equilibrium $\left(\left\{c^{i}\right\}_{i \in I}, p\right)$ and normalise prices so that $\sum_{s \in S} p(s)=1$.

Risk aversion implies that $c^{i}$ has to be constant across all states. If it is not, then consuming $\sum_{s \in S} p(s) c^{i}(s)$ at each state is affordable and weakly better, because $\sum_{s \in S} p(s) u^{i}\left(c^{i}(s)\right) \leq$ $u^{i}\left(\sum_{s \in S} p(s) c^{i}(s)\right)$, from the concavity of $u^{i}$. Moreover, it clears the markets, because from no aggregate uncertainty we have $\sum_{i \in I} \sum_{s \in S} p(s) c^{i}(s)=\sum_{s \in S} p(s) \sum_{i \in I} c^{i}(s)=\omega \sum_{s \in S} p(s)=\omega$. Hence, in equilibrium each risk averse agent is fully insured. From the first order conditions of the maximization problem, we have that $\frac{\pi_{S}(s) u^{\prime \prime}(c(s))}{\pi_{S}\left(s^{\prime}\right) u^{i}\left(c\left(s^{\prime}\right)\right)}=\frac{p(s)}{p\left(s^{\prime}\right)}$ for all $s, s^{\prime} \in S$. Because $c^{i}$ is constant across states, $u^{i \prime}\left(c^{i}(s)\right)=u^{i \prime}\left(c^{i}\left(s^{\prime}\right)\right)$ and therefore $p(s)=\pi_{S}(s)$ for all $s \in S$. In the second example there are risk neutral agents that fully insure the risk averse agents, hence the same arguments apply. 


\subsection{Trading without aggregate uncertainty}

There are two risk averse agents, equipped with the same utility function $u(x)=x-x^{2} / 4$, where $x$ is the amount of the single good that is being consumed. Their endowments are given in Table 1. There is no aggregate uncertainty as the endowments always sum to 1 .

\begin{tabular}{ccc} 
Level of assets & Agent 1 & Agent 2 \\
\hline$a_{h}$ & 1 & 0 \\
$a_{m}$ & 0.5 & 0.5 \\
$a_{l}$ & 0 & 1 \\
\hline
\end{tabular}

Table 1: Endowments

We compare the difference in ex ante expected utility of the two agents in four, successively more informative, structures. In the first, the agents are only aware of question $a$ and they never receive any information about its answer. As the arrows show in Figure 2(a), at each state $s^{*} \in S^{*}$ they are only aware of $S_{0}$ and they consider all three states $\left(a_{h}, a_{m}\right.$ and $\left.a_{l}\right)$ to be possible.

Their posterior over $S_{0}$ is equal to their prior, given by $(0.3,0.3,0.4)$. In all equilibria, the price of the good in the payoff relevant state $s \in S_{0}$ is just the posterior probability of that state. For each $s^{*} \in S^{*}$, the equilibrium allocation is unique. The two risk averse agents insure fully and consume their expected endowment. Therefore, $c^{1}\left(s^{*}\right)=0.45$ and $c^{2}\left(s^{*}\right)=0.55$, for each $s^{*} \in S^{*}$. Agent 1's ex ante expected utility is 0.399 , whereas 2's is 0.474 . Table 2 summarizes the consumption and posteriors for each agent.

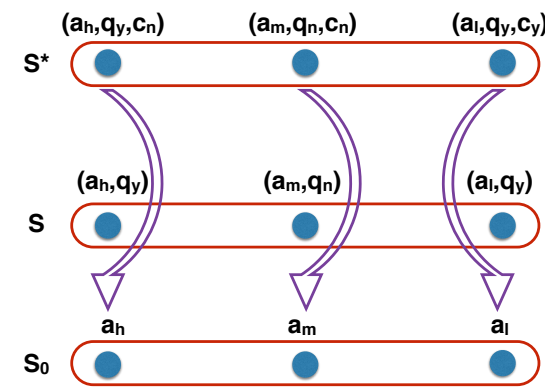

(a) No awareness and no information

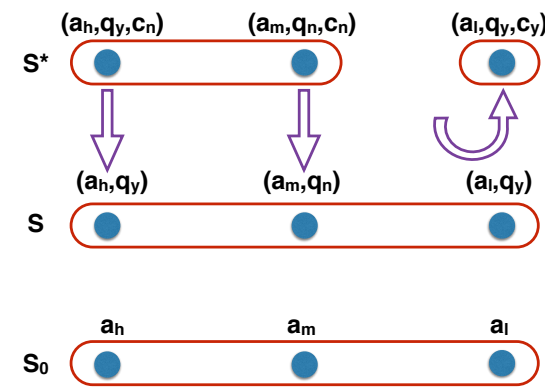

(b) Awareness signal

Figure 2: Information structures 1 and 2

In the second structure, depicted in Figure 2(b), the agents' awareness is not constant across states, but varies. This creates an awareness signal, depicted in each state space by the partition given by the solid lines. At $s_{1}^{*}=\left(a_{h}, q_{y}, c_{n}\right)$ and $s_{2}^{*}=\left(a_{m}, q_{n}, c_{n}\right)$, they are aware of questions $a$ and $q$ and their state space is $S$. At $s_{3}^{*}=\left(a_{l}, q_{y}, c_{y}\right)$, the housing market crisis occurs and they additionally become aware of dimension $c$. Their state space is now $S^{*}=\left\{s_{1}^{*}, s_{2}^{*}, s_{3}^{*}\right\}$. They never receive any information about the answers of the questions they are aware of.

If the agents are aware of all three questions and their state space is $S^{*}$, they can understand that their awareness signal partitions $S^{*}$ into two components, $\left\{\left(a_{h}, q_{y}, c_{n}\right),\left(a_{m}, q_{n}, c_{n}\right)\right\}$ which 


\begin{tabular}{cccc} 
Full state & Posteriors & Agent 1's Consumption & Agent 2's Consumption \\
\hline$s_{1}^{*}$ & $(0.3,0.3,0.4)$ & 0.45 & 0.55 \\
$s_{2}^{*}$ & $(0.3,0.3,0.4)$ & 0.45 & 0.55 \\
$s_{3}^{*}$ & $(0.3,0.3,0.4)$ & 0.45 & 0.55 \\
\hline
\end{tabular}

Table 2: No awareness and no information

\begin{tabular}{cccc} 
Full state & Posteriors & Agent 1's Consumption & Agent 2's Consumption \\
\hline$s_{1}^{*}$ & $(0.3,0.3,0.4)$ & 0.45 & 0.55 \\
$s_{2}^{*}$ & $(0.3,0.3,0.4)$ & 0.45 & 0.55 \\
$s_{3}^{*}$ & $(0,0,1)$ & 0 & 1 \\
\hline
\end{tabular}

Table 3: Awareness signal

specifies awareness of $S$, and $\left\{\left(a_{l}, q_{y}, c_{y}\right)\right\}$ which specifies awareness of $S^{*}$. This is because they understand that they become aware of the housing crisis dimension only when it occurs, therefore deducing that the level of assets is low. However, if they are only aware of $a$ and $q$, their state space is $S$ and their awareness signal is completely uninformative, because they cannot reason symmetrically that as they are unaware of the housing crisis dimension, $\left(a_{l}, q_{y}, c_{y}\right)$ has not occurred.

The information processing error that both agents make is to misuse their awareness signal. This mistake matters for welfare if it is informative, changing their interim beliefs. Conditional Independence given state space $S^{*}$ specifies that interim beliefs do not change when we also condition on the awareness signal described in $S^{*}$. In Figure 2(b) and at state $s_{1}^{*}$, the agent's interim beliefs are $(0.3,0.3,0.4)$. However, if the agent was also informed of the awareness signal described in $S^{*}$, her interim beliefs would be $(1 / 2,1 / 2,0)$, thus violating Conditional Independence.

When $s_{1}^{*}$ or $s_{2}^{*}$ occur, the agents are unaware of $c$ and have no information at all. Their posteriors are identical to their prior, $(0.3,0.3,0.4)$. For both these full states, they have the same consumption as in the no information case. When $s_{3}^{*}$ occurs, they are aware of the full state space $S^{*}$. The awareness signal is informative in $S^{*}$, as it distinguishes between the low awareness states $\left(s_{1}^{*}, s_{2}^{*}\right)$ and the high awareness state $s_{3}^{*}$. Hence, they know that $s_{3}^{*}$ has occurred and consume their endowments, 0 and 1, respectively. Table 3 summarizes the posteriors and consumption for each agent. Agent 1's ex ante utility is 0.239 , whereas agent 2's is 0.584 .

In the third information structure, the agents have the same awareness as in the previous case but additionally always know the answer to question $q$. This signal about $q$ is depicted in Figure 3(a) by the dotted lines. Note that this signal is uninformative in $S_{0}$, because it does not contain question $q$. Conditional Independence given $S^{*}$ is violated because at $\left(a_{h}, q_{y}, c_{n}\right)$ the agent's posterior belief about event $\left(a_{l}, q_{y}\right)$ is $4 / 7$, whereas if we condition also on the awareness signal expressed in $S^{*}$ it is 0 . Intuitively, at $\left(a_{l}, q_{y}, c_{y}\right)$ the agent is aware of $S^{*}$ and can reason that $\left(a_{h}, q_{y}, c_{n}\right)$ is impossible, because it describes that her awareness would be $S$. However, at $\left(a_{h}, q_{y}, c_{n}\right)$ she is aware of $S$ and cannot reason that $\left(a_{l}, q_{y}\right)$ is impossible, because this event happens only when her awareness is strictly higher.

When $s_{1}^{*}$ occurs, the agents are unaware of $c$ but can exclude $a_{m}$, as they know the answer to question $q$ is $y$. Their posterior on $S_{0}$ is $(3 / 7,0,4 / 7)$. They consume their expected endowment, which is $(3 / 7) 1+(4 / 7) 0=3 / 7$ for agent 1 and $(3 / 7) 0+(4 / 7) 1=4 / 7$ for agent 2 . At $s_{2}^{*}$, they know that the answer to question $q$ is $n$, which implies that the level of assets is $m$. Hence, both agents consume their endowment, which is 0.5 . At $s_{3}^{*}$, as in the second information structure, they are fully aware and use their awareness signal to deduce that the level of assets is low. 


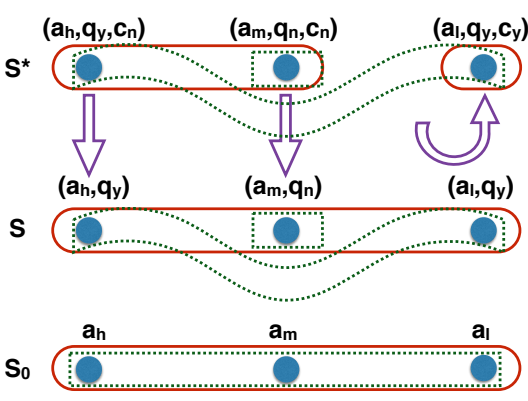

(a) Signal about $q$

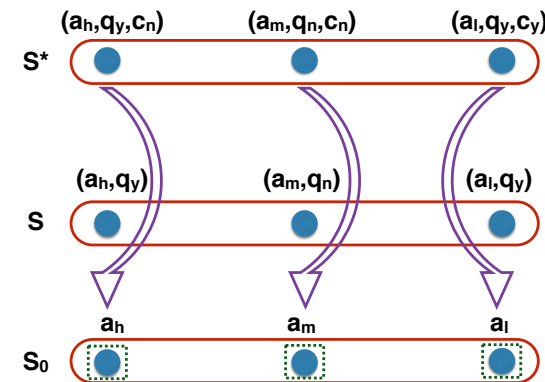

(b) Full information

Figure 3: Information structures 3 and 4

\begin{tabular}{cccc} 
Full state & Posteriors & Agent 1's Consumption & Agent 2's Consumption \\
\hline$s_{1}^{*}$ & $(3 / 7,0,4 / 7)$ & $3 / 7$ & $4 / 7$ \\
$s_{2}^{*}$ & $(0,1,0)$ & 0.5 & 0.5 \\
$s_{3}^{*}$ & $(0,0,1)$ & 0 & 1 \\
\hline
\end{tabular}

Table 4: Signal about $q$

As a result, both consume their endowments, 0 and 1 , respectively. Table 4 summarizes the posteriors and consumption for each agent. Agent 1's ex ante expected utility is 0.246 , whereas 2 's is 0.578 .

In the fourth information structure, depicted in Figure 3(b), the agents have full information, as they are informed of the level of assets before they trade. The signal about $a$ is fully informative, depicted by the discontinuous lines. Their awareness signal is irrelevant, so we assume that they are aware only of the level of assets dimension. Therefore, at each state they consume their endowment. Agent 1's ex ante expected utility is 0.356, whereas 2's is 0.431.

Figure 4 depicts the ex ante expected utilities as information increases. The standard model of Schlee [2001], without unawareness, prescribes that the graphs for both agents should be (weakly) decreasing. However, with unawareness they move in opposite directions. Going from no information to an informative awareness signal, they are willing to insure each when the level of assets is high or medium, not knowing whether a low level of assets has occurred. But when the level of assets is low, their awareness increases and they are able to deduce that the level of assets is low. As a result, the risk-sharing opportunity vanishes, benefiting agent 2 who holds all of the economy's endowment.

Surprisingly, it is not the case that information is always good for agent 2, even though she holds all of the economy's endowment in the state where their awareness increases. If, in addition to the awareness signal, the agents have access to a signal that reveals the answer to question $q$, the roles are reversed: agent 1 becomes better off, whereas agent 2 becomes worse off. Intuitively, knowing that the level of assets is medium at $s_{2}^{*}$ destroys the risk-sharing opportunity in that state, forcing both agents to consume their endowment. Because agent 1 was mistakenly sacrificing some of her endowment at that state to get higher consumption in the event that the level of assets was low, she becomes better off as a result, and the exact 


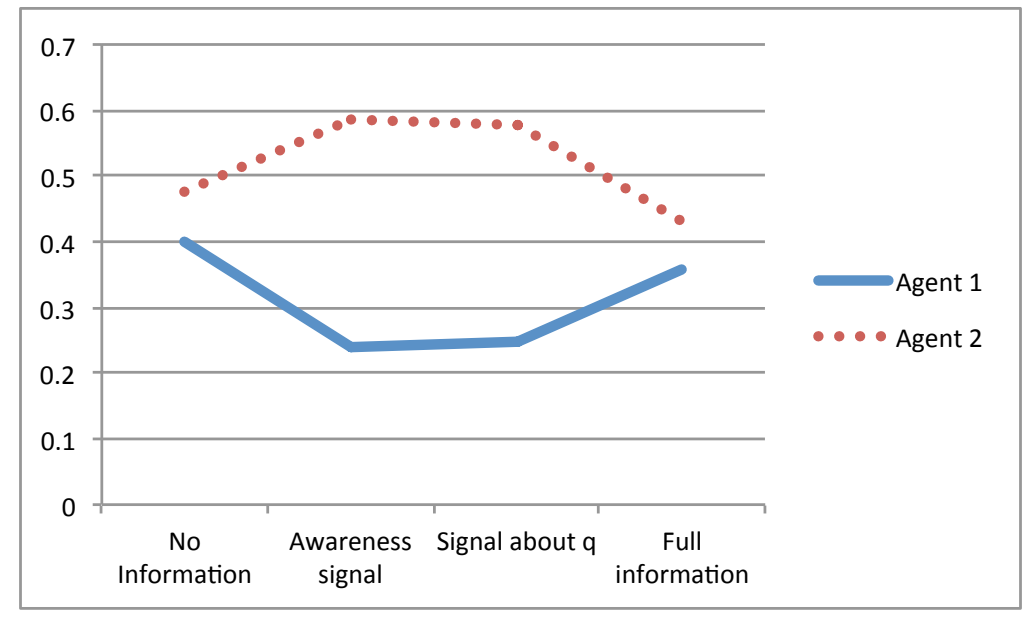

Figure 4: Ex ante utilities as information increases

opposite holds for agent 2.

A similar reasoning explains why moving to full information benefits agent 1 even more, again at the expense of agent 2. The risk-sharing opportunity of insuring when the level of assets is high, for the event that the level of assets is low, is destroyed by more information. However, this is good news for agent 1, because when the level of assets was low, she was not consuming anything anyway. ${ }^{12}$

This example shows that in Theorem 1 we need both $P^{1}$ and $P^{2}$ to satisfy Conditional Independence for the value of information to be negative for all. In particular, if $P^{1}$ is the no information structure and $P^{2}$ is the structure with the awareness signal, then only $P^{1}$ satisfies Conditional Independence and agent 2 becomes strictly better off. If $P^{1}$ is information structure 3 and $P^{2}$ is information structure 4, then only $P^{2}$ satisfies Conditional Independence but agent 1 becomes strictly better off. In Section 3.2 we provide a similar counterexample for the case of risk neutral agents who insure the risk averse agents fully.

It is noteworthy that, unlike the example of Figure 1, here the full state space $S^{*}$ does not consist of the Cartesian product of all the possible answers for each question. For example, full states $\left(a_{h}, q_{y}, c_{y}\right),\left(a_{h}, q_{n}, c_{y}\right),\left(a_{m}, q_{y}, c_{y}\right)$ and $\left(a_{m}, q_{n}, c_{y}\right)$ do not belong to $S^{*}$, implying that if the agent is aware of $S^{*}$ and knows that there is a housing market crisis, she can deduce that the level of the assets is low. ${ }^{13}$ In other words, $S^{*}$ contains connections between answers of different questions, or theorems.

However, these connections are not what drives the violation of Conditional Independence and hence the positive value of public information. To see this, suppose we enlarge the information structure of Figure 3(a), by defining $S^{C}$ to be the Cartesian product of all the possible answers. State spaces $S$ and $S_{0}$ are similarly expanded. The agent still receives the signal about $q$ when she is aware of this dimension, but all states $s^{*} \in S^{C} \backslash S^{*}$ specify that the agent is aware only of $S_{0}$ and therefore has no information. Then, even if these extra states have positive prior probability, Conditional Independence is still violated in the same way as before. For example, consider a uniform prior on the 12 states of $S^{C}$. At $\left(a_{h}, q_{y}, c_{n}\right)$, the agent's posterior belief about event $\left(a_{l}, q_{y}\right)$ is $1 / 2$, whereas if we condition also on the awareness signal expressed in $S^{C}$ it is 0 . The reason is that at each $s^{*} \in S^{*}, S_{0} \prec S^{i}\left(s^{*}\right)$ implies that she considers the event

\footnotetext{
${ }^{12}$ Note that if we compare no information with full information then awareness is constant, so the ex ante utilities for both agents decrease, as predicted in Schlee [2001].

${ }^{13}$ Recall that the formal model of Section 2 only specifies a set of state spaces, not a collection of questions and answers, hence it allows for such a formulation.
} 
$\left(S^{C} \backslash S^{*}\right)_{S^{i}\left(s^{*}\right)}$ to be impossible, because it describes a strictly lower awareness. Therefore, whether Conditional Independence is satisfied or not does not depend on whether $S^{C} \backslash S^{*}$ is included in the information structure.

Moreover, Heifetz et al. [2006, 2013] also allow for information structures where the full state space is not the Cartesian product of all possible answers. However, Proposition 1 shows that their Projections Preserve Posteriors property implies Conditional Independence. Intuitively, Projections Preserve Posteriors can be violated in two different ways. The first, described right after Proposition 1, satisfies Conditional Independence and requires a state space that is not a Cartesian product, because it exploits the connections betweens answers of different questions. The second, described here, does not require a Cartesian product but violates Conditional Independence. On the other hand, the state space in Karni and Vierø [2013] has the form of a Cartesian product, as it is the set of all functions from acts to consequences.

Finally, note that information structures 2 and 3 are extreme in the sense that the agents become aware of all three dimensions if and only if $a_{l}$ (or, equivalently, $c_{y}$ ) occurs. The justification is that the housing crisis is such a dramatic event that increases the awareness of the agents. However, a less extreme assumption would be to specify that when an agent becomes aware of a new dimension, she entertains the possibility that she would have become aware of it under other circumstances, for example if $a_{h}$ or $a_{m}$ (or, equivalently, $c_{n}$ ) had occurred. ${ }^{14}$

This can be modelled by $S^{*}$ having six states, the original three plus their copies, so that $\left(a_{h}, q_{y}, c_{n}\right)_{1}$ specifies that the agents' awareness is $S$, whereas $\left(a_{h}, q_{y}, c_{n}\right)_{2}$ specifies that their awareness is $S^{*}$, and similarly for $\left(a_{m}, q_{n}, c_{n}\right)_{k}$ and $\left(a_{l}, q_{y}, c_{y}\right)_{k}$, where $k=1,2$. The other two state spaces remain the same. It is still possible to provide counterexamples with positive value of information for some agent, as long as Conditional Independence is violated. In information structure 2, when the agent is only aware of $S$ she has no other information, hence she considers all states in $S$ to be possible. Her beliefs about event $\left\{a_{l}\right\}$ are not updated and they are equal to $\pi_{S}\left(\left\{a_{l}\right\}^{S}\right)=\pi_{S^{*}}\left(\left\{\left(a_{l}, q_{y}, c_{y}\right)_{1},\left(a_{l}, q_{y}, c_{y}\right)_{2}\right\}\right)$. When she is aware of $S^{*}$, she knows that the event "I am aware of $S^{*}$ ", denoted $\left\{\left(a_{h}, q_{y}, c_{n}\right)_{2},\left(a_{m}, q_{n}, c_{n}\right)_{2},\left(a_{l}, q_{y}, c_{y}\right)_{2}\right\}$, has occurred. Conditional Independence is violated if, $\pi_{S^{*}}\left(\left\{\left(a_{l}, q_{y}, c_{y}\right)_{1},\left(a_{l}, q_{y}, c_{y}\right)_{2}\right\}\right) \neq \frac{\pi_{S^{*}}\left(\left(a_{l}, q_{y}, c_{y}\right)_{2}\right)}{\pi_{S^{*}}\left(\left\{\left(a_{h}, q_{y}, c_{n}\right)_{2},\left(a_{m}, q_{n}, c_{n}\right)_{2},\left(a_{l}, q_{y}, c_{y}\right)_{2}\right\}\right)}$. For instance, if the right hand side is higher, there is positive correlation between being fully aware and $\left\{a_{l}\right\}$ occurring.

\subsubsection{Asymmetric awareness}

Consider now an example with asymmetric awareness, which shows that it is possible for an agent to intentionally make some of her awareness and information public, in order to manipulate the other agent. Agent 2 (hedge fund manager) is aware of all three questions ex ante, whereas agent 1 is only aware of $a$. Hence, their awareness signals are uninformative. First, suppose that both have no signals about the questions, so they will trade and their consumptions will be identical to those of the first information structure, given in Table 2. Agent 1's ex ante expected utility is 0.399 , whereas 2 's is 0.474 .

Second, suppose that agent 2 is able to acquire signals about $q$ and $c$, which enables her to know what is the level of assets at each state. Her information structure is depicted in Figure $5(\mathrm{~b})$. Note that whereas $S^{*}$ specifies that 2 is able to distinguish between all states, $S$ only describes that she knows whether the level of assets is medium or not, because it does not contain dimension $c$. Moreover, $S_{0}$ describes that 2 has no information. Agent 1 , who is only aware of $S_{0}$, is therefore unaware that agent 2 can know what is the level of assets, because she is unaware of both $q$ and $c$.

Agent 2 can increase her ex ante expected utility by making agent 1 aware of (and providing information about) dimension $q$ and by making agent 1 aware of dimension $c$ only when there

\footnotetext{
${ }^{14}$ I thank a referee for pointing this out.
} 
is a housing crisis and the level of assets is low. In that case, agent 1's information structure is depicted in Figure 3(a).

At state $s_{1}^{*}$, the public information reveals that the level of assets is either high or medium. Agent 2 knows that the level of assets is high, but she has no incentive of revealing it, because her endowment at that state is 0 . Agent 1, being unaware of $c$, thinks that agent 2's information is identical to hers. At state $s_{2}^{*}$, the public information reveals that the level of assets is medium, so both agents consume their endowments. At $s_{3}^{*}$, agent 2 learns the answer to both $q$ and $c$ and deduces that the level of assets is low. She makes agent 1 fully aware, who then realizes that this happens if and only if the level of assets is low. Hence, both consume their endowments. The consumptions of the agents are identical to the case of information structure 3 , given in Table 4. Agent 1's ex ante expected utility is 0.246 , whereas 2's is 0.578 . Comparing with the case of no information, agent 2 is strictly better off by intentionally making some of her private information public and making agent 1 more aware. Moreover, agent 2's plan is dynamically consistent, because she has no incentive in the interim stage to reveal more or less information than what she had planned in the ex ante stage.

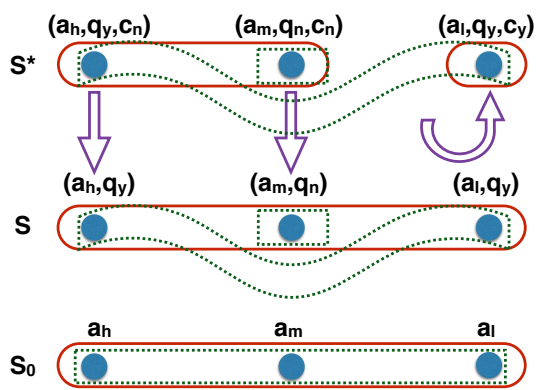

(a) Signal about $q$

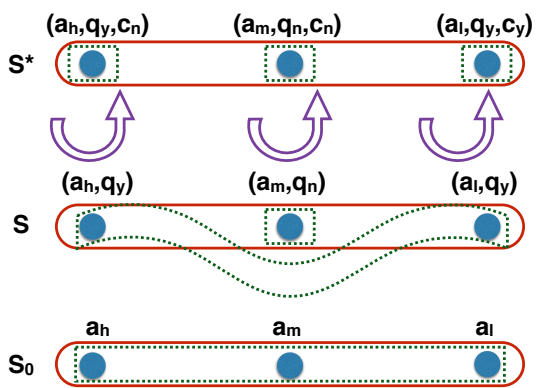

(b) Full awareness and signal about $q$ and $c$

Figure 5: Asymmetric information structures of agents 1 and 2

\subsection{Trading with aggregate uncertainty}

We now show that the value of information is strictly positive for a risk averse agent, in the case where there is aggregate uncertainty and there is a third, risk neutral agent with utility $u(c)=c$, who can insure the other two, risk averse agents. The endowments are given in the following table.

\begin{tabular}{cccc} 
Level of assets & Agent 1 & Agent 2 & Agent 3 \\
\hline$a_{h}$ & 1 & 0 & 0.8 \\
$a_{m}$ & 1 & 0.8 & 0 \\
$a_{l}$ & 1.4 & 1 & 1 \\
\hline
\end{tabular}

The utilities for the two risk averse agents and the common prior are the same as in the previous example. We compare the ex ante expected utilities of the three agents, for the first four information structures described in the previous section.

In the no information case, the posterior of the agents is their prior. The two risk averse agents insure fully, so $c^{1}(s)=1.16$ and $c^{2}(s)=0.64$, for each $s \in S_{0}$. Agent 3 is risk neutral 
and puts all her consumption at state 3, which has the highest probability. Her consumption is 0 at $s_{1}, s_{2}$ and 1.6 at $s_{3}$. Agent 1's ex ante expected utility is $0.823,2$ 's is 0.537 and 3's is 0.64 .

In the awareness signal case, the posteriors are the same as in Table 3. The consumption vectors for the three agents are $c^{1}=\{1.16,1.16,1.4\}, c^{2}=\{0.64,0.64,1\}$ and $c^{3}=\{0,0,1\}$. Agent 1's ex ante expected utility is $0.858,2$ 's is 0.622 and 3's is 0.4 .

When the signal about $q$ is available, the posteriors are the same as in Table 4 . The consumption vectors for the three agents are $c^{1}=\{1.22,1,1.4\}, c^{2}=\{0.57,0.8,1\}$ and $c^{3}=\{0,0,1\}$. Agent 1's ex ante expected utility is $0.844,2$ 's is 0.638 and 3 's is 0.4 .

In the full information case, all agents always consume their endowments. Agent 1's ex ante expected utility is $0.814,2$ 's is 0.492 and 3's is 0.64 . The ex ante utilities are depicted in Figure 6. Note that, unlike the previous example, 1's and 2's ex ante utilities do not always move in opposite directions.

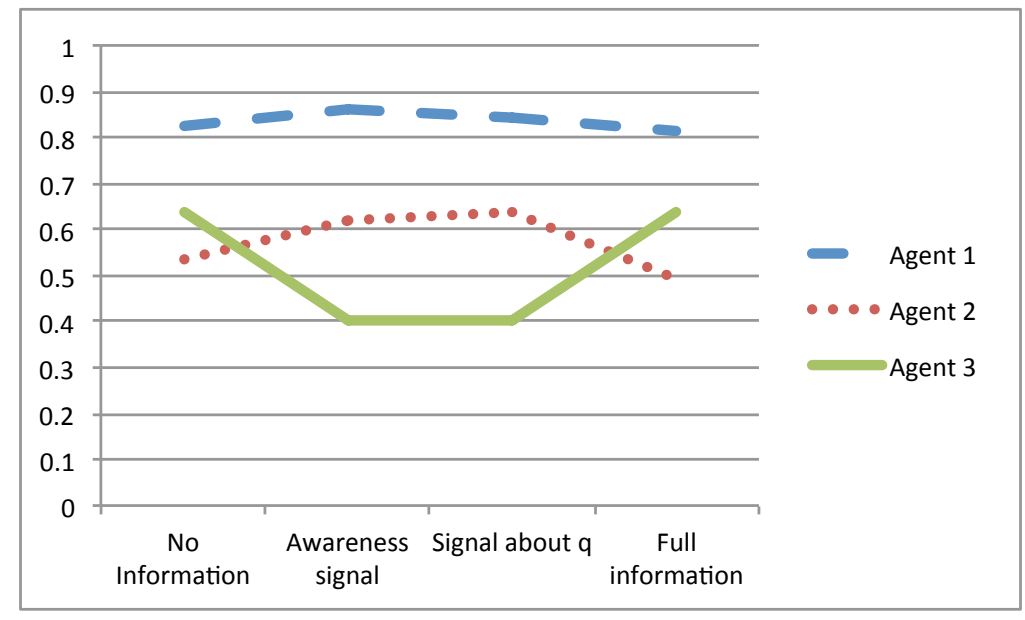

Figure 6: Ex ante utilities as information increases

Finally, as with the asymmetric awareness case that we examine in the previous example, suppose that agents 1 and 3 are unaware and uninformed, as described in Figure 2(a). Then, a fully aware but uninformed agent 2 will be strictly better off by acquiring information about $q$ and $c$, then revealing some of her awareness and information to agents 1 and 3 , so that their information structure is described by Figure 3(a).

\section{A Proofs}

Proof of Proposition 1. Fix $s \in S \in \mathcal{S}$ with $\pi_{S}(s)>0$ and event $E \subseteq S^{i}(s)$. We need to show that $\pi_{S^{i}(s)}\left(E \mid P^{i}(s)\right)=\pi_{S}\left(E^{S} \mid \mathcal{E}_{S}^{i}(s) \cap P^{i}(s)^{S}\right)=m$. If $P^{i}(s)^{S} \subseteq \mathcal{E}_{S}^{i}(s)$ then we are done, so suppose that $P^{i}(s)^{S} \nsubseteq \mathcal{E}_{S}^{i}(s)$.

First, we show that if $s_{1} \in P^{i}(s)^{S}$ then $S^{i}\left(s_{1}\right) \succeq S^{i}(s), P^{i}\left(s_{1}\right)^{S} \subseteq P^{i}(s)^{S}$ and $\pi_{S^{i}\left(s_{1}\right)}\left(E^{S^{i}\left(s_{1}\right)} \mid P^{i}\left(s_{1}\right)\right)=$ $m$. Generalized Reflexivity and Stationarity imply that $P^{i}\left(\left\{s_{1}\right\}_{S^{i}(s)}\right)=P^{i}(s)$. Projections Preserve Ignorance implies that $S^{i}\left(s_{1}\right) \succeq S^{i}(s)$ and $P^{i}\left(s_{1}\right)^{S} \subseteq P^{i}\left(\left\{s_{1}\right\}_{S^{i}(s)}\right)^{S}=P^{i}(s)^{S}$. Moreover, $S^{i}\left(s_{1}\right) \succeq S^{i}(s)$ and Projections Preserve Posteriors imply that $\pi_{S^{i}\left(s_{1}\right)}\left(E^{S^{i}\left(s_{1}\right)} \mid P^{i}\left(s_{1}\right)\right)=$ $\pi_{S^{i}\left(\left\{s_{1}\right\}_{S^{i}(s)}\right)}\left(E^{S^{i}\left(\left\{s_{1}\right\}_{S^{i}(s)}\right)} \mid P^{i}\left(\left\{s_{1}\right\}_{S^{i}(s)}\right)\right)=\pi_{S^{i}(s)}\left(E \mid P^{i}(s)\right)=m$.

Second, we show that $P^{i}(s)^{S}$ is partitioned by sets of the form $\mathcal{E}_{S}^{i}\left(s_{1}\right) \cap P^{i}\left(s_{1}\right)^{S}$, where $s_{1} \in P^{i}(s)^{S}$. From the previous paragraph, $s_{1} \in P^{i}(s)^{S}$ implies $P^{i}\left(s_{1}\right)^{S} \subseteq P^{i}(s)^{S}$, therefore $P^{i}\left(s_{1}\right)^{S} \cap \mathcal{E}_{S}^{i}\left(s_{1}\right) \subseteq P^{i}(s)^{S}$. From Generalized Reflexivity, $s_{1} \in P^{i}\left(s_{1}\right)^{S} \cap \mathcal{E}_{S}^{i}\left(s_{1}\right)$. Suppose $s_{2} \in$ 
$P^{i}\left(s_{1}\right)^{S} \cap \mathcal{E}_{S}^{i}\left(s_{1}\right)$. Then, $S^{i}\left(s_{2}\right)=S^{i}\left(s_{1}\right)$, which implies $\mathcal{E}_{S}^{i}\left(s_{1}\right)=\mathcal{E}_{S}^{i}\left(s_{2}\right)$, and $\left\{s_{2}\right\}_{S^{i}\left(s_{1}\right)} \in P^{i}\left(s_{1}\right)$. Moreover, Generalized Reflexivity implies that $\left\{s_{2}\right\}_{S^{i}\left(s_{1}\right)} \in P^{i}\left(s_{2}\right)$. Thus, from Stationarity, it follows that $P^{i}\left(s_{2}\right)=P^{i}\left(\left\{s_{2}\right\}_{S^{i}\left(s_{1}\right)}\right)=P^{i}\left(s_{1}\right)$. Hence, $P^{i}\left(s_{2}\right)^{S} \cap \mathcal{E}_{S}^{i}\left(s_{2}\right)=P^{i}\left(s_{1}\right)^{S} \cap \mathcal{E}_{S}^{i}\left(s_{1}\right)$.

Define $U_{1}=\left\{t \in P^{i}(s)^{S}: \nexists s^{\prime} \in P^{i}(s)^{S}\right.$ such that $\left.S^{i}\left(s^{\prime}\right) \succ S^{i}(t)\right\}$ and, for $j>1, U_{j}=\{t \in$ $P^{i}(s)^{S}: \nexists s^{\prime} \in P^{i}(s)^{S} \backslash\left(U_{1} \cup \ldots \cup U_{j-1}\right)$ such that $\left.S^{i}\left(s^{\prime}\right) \succ S^{i}(t)\right\}$. Let $j=1, \ldots, n$, where $U_{n}$ is the last non empty such set. Because $S$ is finite, $n$ is finite as well. We prove the claim using induction on $j$.

Let $s_{1} \in U_{1}$. Suppose $s_{2} \in P^{i}\left(s_{1}\right)^{S}$. Using the previous arguments, $S^{i}\left(s_{2}\right) \succeq S^{i}\left(s_{1}\right)$. Because $s_{1} \in U_{1}, S^{i}\left(s_{2}\right)=S^{i}\left(s_{1}\right)$ and $s_{2} \in \mathcal{E}_{S}^{i}\left(s_{1}\right)$. Therefore, $\mathcal{E}_{S}^{i}\left(s_{1}\right) \cap P^{i}\left(s_{1}\right)^{S}=P^{i}\left(s_{1}\right)^{S}$ and $\pi_{S}\left(E^{S} \mid \mathcal{E}_{S}^{i}\left(s_{1}\right) \cap P^{i}\left(s_{1}\right)^{S}\right)=\pi_{S^{i}\left(s_{1}\right)}\left(E^{S^{i}\left(s_{1}\right)} \mid P^{i}\left(s_{1}\right)\right)=m$.

Suppose that for all $j \leq k, s_{1} \in U_{j}$ implies that $\pi_{S}\left(E^{S} \mid \mathcal{E}_{S}^{i}\left(s_{1}\right) \cap P^{i}\left(s_{1}\right)^{S}\right)=\pi_{S^{i}\left(s_{1}\right)}\left(E^{S^{i}\left(s_{1}\right)} \mid P^{i}\left(s_{1}\right)\right)=$ $m$. Let $j=k+1$ and suppose $s_{1} \in U_{j}$. Let $s_{2} \in P^{i}\left(s_{1}\right)^{S}$. From previous arguments, $S^{i}\left(s_{2}\right) \succeq S^{i}\left(s_{1}\right)$ and $P^{i}\left(s_{2}\right)^{S} \subseteq P^{i}\left(s_{1}\right)^{S}$. Moreover, if $S^{i}\left(s_{2}\right) \succ S^{i}\left(s_{1}\right)$ then $s_{2} \in U_{j}$ for $j<k+1$. We partition $P^{i}\left(s_{1}\right)^{\bar{S}}$ with sets of the form $\mathcal{E}_{S}^{i}\left(s_{2}\right) \cap P^{i}\left(s_{2}\right)^{S}$, where $s_{2} \in P^{i}\left(s_{1}\right)^{S}$. For each element $\mathcal{E}_{S}^{i}\left(s_{2}\right) \cap P^{i}\left(s_{2}\right)^{S}$ of that partition which is distinct from $\mathcal{E}_{S}^{i}\left(s_{1}\right) \cap P^{i}\left(s_{1}\right)^{S}$, we have $s_{2} \in U_{j}$, for some $j \leq k$ and from the induction hypothesis we have $\pi_{S}\left(E^{S} \mid \mathcal{E}_{S}^{i}\left(s_{2}\right) \cap\right.$ $\left.P^{i}\left(s_{2}\right)^{S}\right)=\pi_{S^{i}\left(s_{2}\right)}\left(E^{S^{i}\left(s_{2}\right)} \mid P^{i}\left(s_{2}\right)\right)=m$. Let $B$ be the union of these elements. Then we have $\pi_{S}\left(E^{S} \mid B\right)=m$, which by Projections Preserve Posteriors implies

$$
\begin{gathered}
\pi_{S}\left(E^{S} \mid \mathcal{E}_{S}^{i}\left(s_{1}\right) \cap P^{i}\left(s_{1}\right)^{S}\right)=\frac{\pi_{S}\left(E^{S} \cap \mathcal{E}_{S}^{i}\left(s_{1}\right) \cap P^{i}\left(s_{1}\right)^{S}\right)}{\pi_{S}\left(\mathcal{E}_{S}^{i}\left(s_{1}\right) \cap P^{i}\left(s_{1}\right)^{S}\right)}=\frac{\pi_{S}\left(E^{S} \cap P^{i}\left(s_{1}\right)^{S}\right)-\pi_{S}\left(E^{S} \cap B\right)}{\pi_{S}\left(P^{i}\left(s_{1}\right)^{S}\right)-\pi_{S}(B)}= \\
=\frac{m\left(\pi_{S}\left(P^{i}\left(s_{1}\right)^{S}\right)-\pi_{S}(B)\right)}{\pi_{S}\left(P^{i}\left(s_{1}\right)\right)-\pi_{S}(B)}=m=\pi_{S^{i}\left(s_{1}\right)}\left(E \mid P^{i}\left(s_{1}\right)\right) .
\end{gathered}
$$

Proof of Proposition 2. Let $c_{S, P^{1}}$ be the resulting allocation from the equilibrium in economy $\left(S, P^{1},\left\{\omega^{i}\right\}_{i \in I},\left\{u^{i}\right\}_{i \in I}, \pi\right)$ and $d_{S, P^{2}}$ the resulting allocation from the equilibrium in economy $\left(S, P^{2},\left\{\omega^{i}\right\}_{i \in I},\left\{u^{i}\right\}_{i \in I}, \pi\right)$. Note that, since $P^{1}$ is uninformative, $c_{S, P^{1}}^{i}$ is constant conditional on each payoff relevant state $s_{0} \in S_{0}$, for each $i \in I$.

For all $i \in I$, for each $s_{0} \in S_{0}$, from the concavity of $u^{i}$ we have

$$
\sum_{s \in s_{0}^{S}} \frac{\pi_{S}(s)}{\pi_{S}\left(s_{0}^{S}\right)} u^{i}\left(d_{s}^{i}\left(s_{S^{2}(s)}\right)\right) \leq u^{i}\left(\sum_{s \in s_{0}^{S}} \frac{\pi_{S}(s)}{\pi_{S}\left(s_{0}^{S}\right)} d_{s}^{i}\left(s_{S^{2}(s)}\right)\right),
$$

with strict inequality if $u^{i}$ is strictly concave and $d_{S, P^{2}}^{i}$ is not constant conditional on $s_{0}$. If we take the expectation with respect to all $s_{0} \in S_{0}$, we have

$$
\begin{gathered}
\sum_{s_{0} \in S_{0}} \pi_{S}\left(s_{0}^{S}\right) \sum_{s \in s_{0}^{S}} \frac{\pi_{S}(s)}{\pi_{S}\left(s_{0}^{S}\right)} u^{i}\left(d_{s}^{i}\left(s_{S^{2}(s)}\right)\right) \leq \sum_{s_{0} \in S_{0}} \pi_{S}\left(s_{0}^{S}\right) u^{i}\left(\sum_{s \in s_{0}^{S}} \frac{\pi_{S}(s)}{\pi_{S}\left(s_{0}^{S}\right)} d_{s}^{i}\left(s_{S^{2}(s)}\right)\right) \Longrightarrow \\
\sum_{s \in S} \pi_{S}(s) u^{i}\left(d_{s}^{i}\left(s_{S^{2}(s)}\right)\right) \leq \sum_{s_{0} \in S_{0}} \pi_{S}\left(s_{0}^{S}\right) u^{i}\left(\sum_{s \in s_{0}^{S}} \frac{\pi_{S}(s)}{\pi_{S}\left(s_{0}^{S}\right)} d_{s}^{i}\left(s_{S^{2}(s)}\right)\right) .
\end{gathered}
$$

Because $P^{1}$ is uninformative given $S$, the agents' awareness and their state space is the same for all $s \in S$. Let $S^{1}$ be the agents' state space. Then, for all $s \in S, P^{1}(s)=S^{1}$. Define the following allocation $g_{S, P^{1}}$. Because $P^{1}$ is uninformative, $g_{s_{1}}^{i}=g_{s_{2}}^{i}$ for all $s_{1}, s_{2} \in S$, so we only need to define it for some $s \in S$. For each $s_{0} \in S_{0}$, for all $s_{1} \in s_{0}^{S^{1}}$, let $g_{s}^{i}\left(s_{1}\right)=$ 
$\sum_{s^{\prime} \in\left\{s_{1 S_{0}}\right\}^{S}} \frac{\pi_{S}\left(s^{\prime}\right)}{\pi_{S}\left(\left\{s_{1 S_{0}}\right\}^{S}\right)} d_{s^{\prime}}^{i}\left(s^{\prime} S^{2}\left(s^{\prime}\right)\right)$. This allocation specifies that, if $s_{1} \in S^{1}$ occurs, $i$ will get the average of what she would get under $d_{S, P^{2}}$, given that the payoff relevant state $s_{1 S_{0}}$ (the projection of $s_{1}$ to $\left.S_{0}\right)$ has occurred. Hence, if $s_{2}, s_{3} \in s_{0}^{S}$ for some $s_{0} \in S_{0}$, then $g_{s_{2}}^{i}\left(s_{2 S^{1}}\right)=$ $g_{s_{3}}^{i}\left(s_{3 S^{1}}\right)$. Because projections commute, we have that, for each $s \in S,\left\{s_{S^{1}}\right\}_{S_{0}}=s_{S_{0}}$ and $g_{s}^{i}\left(s_{S^{1}}\right)=\sum_{s^{\prime} \in\left\{s_{S_{0}}\right\}^{S}} \frac{\pi_{S}\left(s^{\prime}\right)}{\pi_{S}\left(\left\{s_{S_{0}}\right\}^{S}\right)} d_{s^{\prime}}^{i}\left(s^{\prime} S^{2}\left(s^{\prime}\right)\right)$, which is equal to the term inside the parenthesis in the right hand side of inequality (1), for $s_{S_{0}} \in S_{0}$. We can then rewrite (1) as

$$
\sum_{s \in S} \pi_{S}(s) u^{i}\left(d_{s}^{i}\left(s_{S^{2}(s)}\right)\right) \leq \sum_{s \in S} \pi_{S}(s) u^{i}\left(g_{s}^{i}\left(s_{S^{1}}\right)\right) .
$$

We next show that $g_{S, P^{1}}$ is feasible. For each $s \in S$, for all $s_{1} \in P^{1}(s)=S^{1}$, we have

$$
\begin{gathered}
\sum_{i \in I} g_{s}^{i}\left(s_{1}\right)=\sum_{i \in I} \sum_{s^{\prime} \in\left\{s_{\left.1 S_{0}\right\}}\right\}^{S}} \frac{\pi_{S}\left(s^{\prime}\right)}{\pi_{S}\left(\left\{s_{\left.\left.1 S_{0}\right\}^{S}\right)}\right.\right.} d_{s^{\prime}}^{i}\left(s^{\prime}{ }_{S^{2}\left(s^{\prime}\right)}\right)= \\
=\sum_{s^{\prime} \in\left\{s_{1 S_{0}}\right\}^{S}} \frac{\pi_{S}\left(s^{\prime}\right)}{\pi_{S}\left(\left\{s_{1 S_{0}}\right\}^{S}\right)} \sum_{i \in I} d_{s^{\prime}}^{i}\left(s^{\prime}{ }_{S^{2}\left(s^{\prime}\right)}\right) \leq \sum_{s^{\prime} \in\left\{s_{1 S_{0}}\right\}^{S}} \frac{\pi_{S}\left(s^{\prime}\right)}{\pi_{S}\left(\left\{s_{\left.\left.1 S_{0}\right\}^{S}\right)} \omega\left(s_{1}\right)=\omega\left(s_{1}\right) .\right.\right.}
\end{gathered}
$$

Since $P^{1}$ is uninformative and specifies constant awareness, $c_{S, P^{1}}$ is ex ante Pareto efficient. In particular, it is ex ante Pareto undominated by $g_{S, P^{1}}$, which is feasible and constant conditional on each payoff relevant state $s_{0} \in S_{0}$. Hence, there exists an agent $i$ such that, for all $s \in S, \sum_{s_{1} \in S^{1}} \pi_{S_{1}}\left(s_{1}\right) u^{i}\left(g_{s}^{i}\left(s_{1}\right)\right) \leq \sum_{s_{1} \in S^{1}} \pi_{S_{1}}\left(s_{1}\right) u^{i}\left(c_{s}^{i}\left(s_{1}\right)\right)$. Because $S \succeq S^{1}$ and $P^{1}$ is uninformative given $S$, we have that for all $s_{2}, s_{3} \in S$, if $s_{2 S^{1}}=s_{3 S^{1}}$, then $g_{s_{2}}^{i}\left(s_{2 S^{1}}\right)=g_{s_{3}}^{i}\left(s_{3 S^{1}}\right)$ and $c_{s_{2}}^{i}\left(s_{2 S^{1}}\right)=c_{s_{3}}^{i}\left(s_{3 S^{1}}\right)$. This implies that we can write the inequality as $\sum_{s \in S} \pi_{S}(s) u^{i}\left(g_{s}^{i}\left(s_{S^{1}}\right)\right) \leq$ $\sum_{s \in S} \pi_{S}(s) u^{i}\left(c_{s}^{i}\left(s_{S^{1}}\right)\right)$. Combining this with inequality (2) we have that $\sum_{s \in S} \pi_{S}(s) u^{i}\left(d_{s}^{i}\left(s_{S^{2}(s)}\right)\right) \leq$ $\sum_{s \in S} \pi_{S}(s) u^{i}\left(c_{s}^{i}\left(s_{S^{1}}\right)\right)$, hence $i$ is weakly worse off ex ante under $d_{S, P^{2}}$ than under $c_{S, P^{1}}$. If $u^{i}$ is strictly concave and $d_{S, P^{2}}^{i}$ is not constant for all $s_{0} \in S_{0}$, then she is strictly worse off ex ante.

To provide a graphical explanation of the proof, consider Figure 7 . Because $P^{1}$ is uninformative, $P^{1}(s)=S^{1}$ for all $s \in S$, which is the second state space from the top. Agent $i$ 's consumption at $s$ is $c_{s}^{i}\left(s_{S^{1}}\right)$. Under $P^{2}$, however, different states in $S$ specify different awareness. State $s$ specifies that her state space is $S^{2}(s)$, which is the third state space from the top, with consumption $d_{s}^{i}\left(s_{S^{2}(s)}\right)$. State $s^{\prime}$ specifies that her state space is $S^{2}\left(s^{\prime}\right)$, which is the fourth state space from the top, with consumption $d_{s^{\prime}}^{i}\left(s_{S^{2}\left(s^{\prime}\right)}^{\prime}\right)$. The thin arrows show that both $s$ and $s^{\prime}$ project to $s_{S_{0}} \in S_{0}$, which is the bottom state space. Hence, $s, s^{\prime} \in\left\{s_{S_{0}}\right\}^{S}$. By taking the average of the consumption described by $d_{S, P^{2}}$, for all states $s^{\prime} \in\left\{s_{S_{0}}\right\}^{S}$, with weights $\frac{\pi_{S}\left(s^{\prime}\right)}{\pi_{S}\left(\left\{s_{S_{0}}\right\}^{S}\right)}$, we construct allocation $g_{S, P^{1}}$. Because $g_{S, P^{1}}$ is feasible in $\left(S, P^{1},\left\{\omega^{i}\right\}_{i \in I},\left\{u^{i}\right\}_{i \in I}, \pi\right)$, Pareto efficiency of $c_{S, P^{1}}$ implies that some $i$ weakly prefers $c_{S, P^{1}}$ over $g_{S, P^{1}}$. Concavity of $u^{i}$ implies that $i$ weakly prefers $g_{S, P^{1}}$ over $d_{S, P^{2}}$, hence the result.

Proof of Theorem 1. At state $s \in S$ and economy $\left(S, P^{1},\left\{\omega^{i}\right\}_{i \in I},\left\{u^{i}\right\}_{i \in I}, \pi\right)$, the agents' state space is $S^{1}(s)$ and their posterior about $s_{1} \in S^{1}(s)$ is $\frac{\pi_{S^{1}(s)}\left(s_{1}\right)}{\pi_{S^{1}(s)}\left(P^{1}(s)\right)}$ if $s_{1} \in P^{1}(s)$ and 0 otherwise. Therefore, we are at a standard general equilibrium model under uncertainty. It is a standard result (we sketch its proof in Section 3) that if all agents are risk averse and there is no aggregate uncertainty, the only equilibrium allocation is such that each agent $i$ consumes her expected endowment, which is $\sum_{s_{1} \in P^{1}(s)} \frac{\pi_{S^{1}(s)}\left(s_{1}\right)}{\pi_{S^{1}(s)}\left(P^{1}(s)\right)} \omega^{i}\left(s_{1}\right)$, where $\omega^{i}\left(s_{1}\right)$ is $i$ 's endowment at $s_{1}$. 


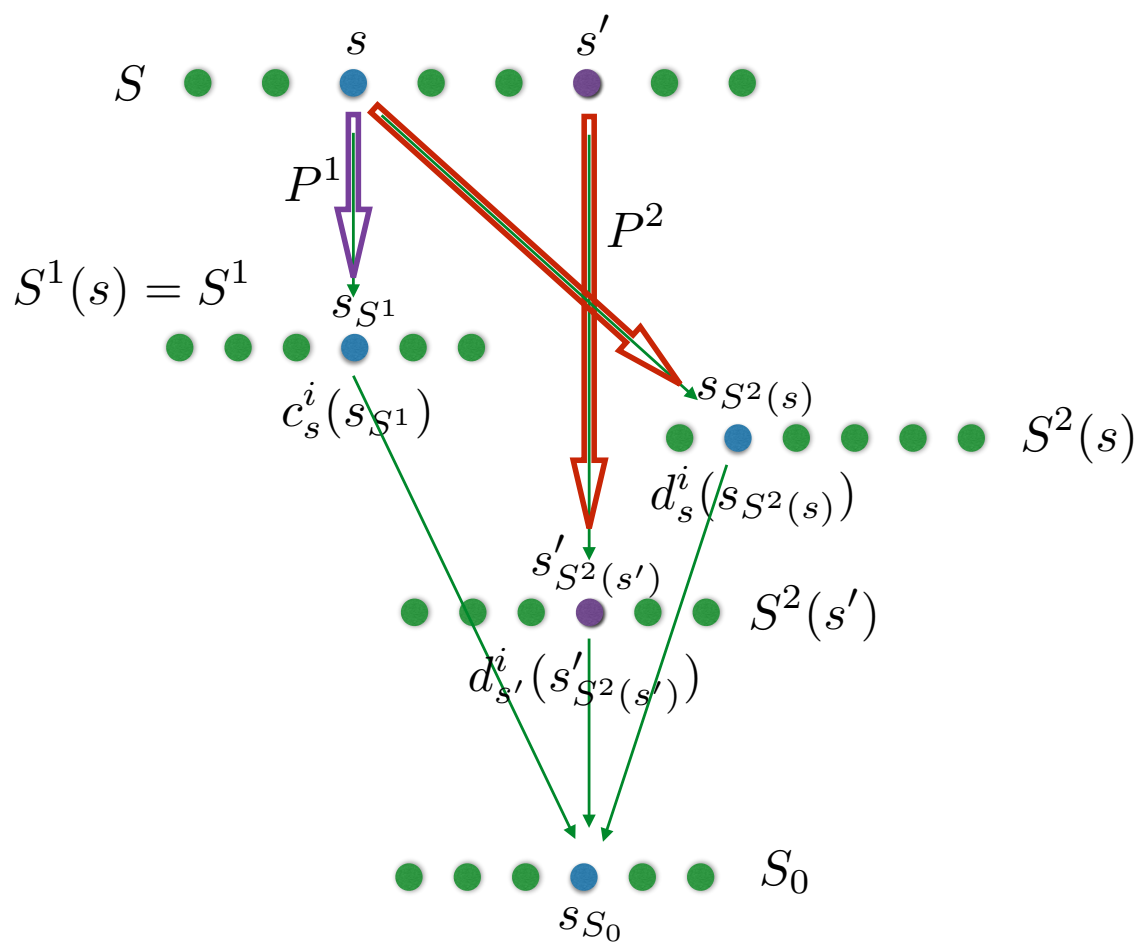

Figure 7: Graphic representation of Proposition 2

In words, take any equilibrium allocation $c_{S, P^{1}}$ and consider the allocation which assigns the expectation $\sum_{s_{1} \in P^{1}(s)} \frac{\pi_{S^{1}(s)}\left(s_{1}\right)}{\pi_{S^{1}(s)}\left(P^{1}(s)\right)} c_{s}^{i}\left(s_{1}\right)$ to each agent $i$. This allocation is feasible because of no aggregate uncertainty and all agents weakly prefer it, because of risk aversion. Moreover, if $i$ 's consumption is not constant across states in the initial allocation, then she strictly prefers the new one. Because consumption is constant across states for all agents, equality of the marginal rates of substitution across states implies that the prices (after normalization) are the posterior probabilities. Therefore, the only equilibrium allocation is such that each agent consumes her expected endowment. Similar arguments apply when there is aggregate uncertainty but there are risk neutral agents who fully insure the risk averse agents. In both cases, agent $i$ 's ex ante expected utility is

$$
\sum_{s \in S} \pi_{S}(s) u^{i}\left(\sum_{s_{1} \in P^{1}(s)} \frac{\pi_{S^{1}(s)}\left(s_{1}\right)}{\pi_{S^{1}(s)}\left(P^{1}(s)\right)} \omega^{i}\left(s_{1}\right)\right) .
$$

Consider the following lemma.

Lemma 1. For any state space $S \in \mathcal{S}$, for any possibility correspondence $P$ and the resulting awareness signal $\mathcal{E}_{S},\left\{P(s)^{S} \cap \mathcal{E}_{S}(s)\right\}_{s \in S}$ is a partition of $S$. Moreover, if $P^{2}$ is more informative than $P^{1}$ given $S \in \mathcal{S}$, then partition $\left\{P^{2}(s)^{S} \cap \mathcal{E}_{S}^{2}(s)\right\}_{s \in S}$ is finer than $\left\{P^{1}(s)^{S} \cap \mathcal{E}_{S}^{1}(s)\right\}_{s \in S}$.

Proof. From Generalized Reflexivity, $s \in P(s)^{S} \cap \mathcal{E}_{S}(s)$. Suppose $s_{1} \in P(s)^{S} \cap \mathcal{E}_{S}(s)$. Then, $S(s)=S\left(s_{1}\right)$ and $\left\{s_{1}\right\}_{S(s)} \in P(s)$. Generalized Reflexivity implies $\left\{s_{1}\right\}_{S(s)} \in P\left(s_{1}\right)$ and 
Stationarity implies $P\left(s_{1}\right)=P\left(\left\{s_{1}\right\}_{S(s)}\right)=P(s)$. Hence, $P\left(s_{1}\right)^{S} \cap \mathcal{E}_{S}\left(s_{1}\right)=P(s)^{S} \cap \mathcal{E}_{S}(s)$.

For the second claim, suppose $s_{1} \in P^{2}(s)^{S} \cap \mathcal{E}_{S}^{2}(s)$. Because $P^{2}$ is more informative than $P^{1}$ given $S$, we have $s_{1} \in P^{1}(s)^{S}$. Stationarity and Projections Preserve Ignorance imply that $S^{1}\left(s_{1}\right) \succeq S^{1}(s)$. Suppose $s_{1} \notin \mathcal{E}_{S}^{1}(s)$. Then, $S^{1}\left(s_{1}\right) \succ S^{1}(s)$, which implies that $s \notin P^{1}\left(s_{1}\right)^{S}$. Because $\left\{P^{2}(s)^{S} \cap \mathcal{E}_{S}^{2}(s)\right\}_{s \in S}$ is a partition, we have $P^{2}(s)^{S} \cap \mathcal{E}_{S}^{2}(s)=P^{2}\left(s_{1}\right)^{S} \cap \mathcal{E}_{S}^{2}\left(s_{1}\right)$, so together with Generalized Reflexivity we have $s \in P^{2}\left(s_{1}\right)^{S} \subseteq P^{1}\left(s_{1}\right)^{S}$, a contradiction.

This lemma says that $\mathbb{P}^{1}=\left\{P^{1}(s)^{S} \cap \mathcal{E}_{S}^{1}(s)\right\}_{s \in S}$ and $\mathbb{P}^{2}=\left\{P^{2}(s)^{S} \cap \mathcal{E}_{S}^{2}(s)\right\}_{s \in S}$ are two partitions of $S$, where $\mathbb{P}^{2}$ is finer than $\mathbb{P}^{1}$. Take a partition cell $\mathbb{P}^{1}(s)=P^{1}(s)^{S} \cap \mathcal{E}_{S}^{1}(s)$, for some $s \in S$. For all states in $\mathbb{P}^{1}(s)$, the agent's awareness is the same. Hence, agent $i$ 's consumption is constant and it is her expected endowment, $\sum_{s_{1} \in P^{1}(s)} \frac{\pi_{S^{1}(s)}\left(s_{1}\right)}{\pi_{S^{1}(s)}\left(P^{1}(s)\right)} \omega^{i}\left(s_{1}\right)$. The expected value of $i$ 's consumption over the partition cell $\mathbb{P}^{1}(s)$ is $\pi_{S}\left(P^{1}(s)^{S} \cap \mathcal{E}_{S}^{1}(s)\right)\left(\sum_{s_{1} \in P^{1}(s)} \frac{\pi_{S^{1}(s)}\left(s_{1}\right)}{\pi_{S^{1}(s)}\left(P^{1}(s)\right)} \omega^{i}\left(s_{1}\right)\right)$. Conditional Independence implies that for every $s \in S$ with $\pi_{S}(s)>0$ and any $s_{1} \in P^{1}(s)$, we have that $\frac{\pi_{S^{1}(s)}\left(s_{1}\right)}{\pi_{S^{1}(s)}\left(P^{1}(s)\right)}=\frac{\pi_{S}\left(s_{1}^{S} \cap \mathcal{E}_{S}^{1}(s)\right)}{\pi_{S}\left(P^{1}(s)^{S} \cap \mathcal{E}_{S}^{1}(s)\right)}$. Substituting, we have that the expected value of $i$ 's consumption over $\mathbb{P}^{1}(s)$ is $\sum_{s_{1} \in P^{1}(s)} \pi_{S}\left(s_{1}^{S} \cap \mathcal{E}_{S}^{1}(s)\right) \omega^{i}\left(s_{1}\right)$. Since $\omega^{i}\left(s_{1}\right)=\omega^{i}\left(s_{2}\right)$ for all $s_{2} \in s_{1}^{S}$, the sum becomes $\sum_{s_{1} \in P^{1}(s)} \sum_{s_{2} \in s_{1}^{S}} \pi_{S}\left(s_{2} \cap \mathcal{E}_{S}^{1}(s)\right) \omega^{i}\left(s_{2}\right)=\sum_{s_{2} \in P^{1}(s)^{S}} \pi_{S}\left(s_{2} \cap \mathcal{E}_{S}^{1}(s)\right) \omega^{i}\left(s_{2}\right)=$ $\sum_{s_{2} \in P^{1}(s)^{S} \cap \mathcal{E}_{S}^{1}(s)} \pi_{S}\left(s_{2} \cap \mathcal{E}_{S}^{1}(s)\right) \omega^{i}\left(s_{2}\right)=\sum_{s_{2} \in \mathbb{P}^{1}(s)} \pi_{S}\left(s_{2}\right) \omega^{i}\left(s_{2}\right)$, because, for each $s_{2} \in \mathbb{P}^{1}(s)$, either $s_{2} \cap \mathcal{E}_{S}^{1}(s)=\emptyset$ or $s_{2} \cap \mathcal{E}_{S}^{1}(s)=s_{2}$.

Using the same arguments as in the previous paragraph, we can show that the expected value of each agent's endowment over $\mathbb{P}^{2}(s)$ is $\sum_{s_{2} \in \mathbb{P}^{2}(s)} \pi_{S}\left(s_{2}\right) \omega^{i}\left(s_{2}\right)$. However, since $\mathbb{P}^{2}$ is finer than $\mathbb{P}^{1}$, an agent in economy $\left(S, P^{2},\left\{\omega^{i}\right\}_{i \in I},\left\{u^{i}\right\}_{i \in I}, \pi\right)$, faces more risk, because her expected endowment is not constant over $\mathbb{P}^{1}(s)$, whereas in economy $\left(S, P^{1},\left\{\omega^{i}\right\}_{i \in I},\left\{u^{i}\right\}_{i \in I}, \pi\right)$ it is constant. Hence, given $\mathbb{P}^{1}(s)$ and from the concavity of $u^{i}$ she weakly prefers the consumption under economy $\left(S, P^{1},\left\{\omega^{i}\right\}_{i \in I},\left\{u^{i}\right\}_{i \in I}, \pi\right)$ than under economy $\left(S, P^{2},\left\{\omega^{i}\right\}_{i \in I},\left\{u^{i}\right\}_{i \in I}, \pi\right)$. Because $\mathbb{P}^{1}$ is a partition, we can extend the results for all the partition cells in $\mathbb{P}^{1}$ and conclude that each agent will be ex ante weakly worse off from the consumption in economy $\left(S, P^{2},\left\{\omega^{i}\right\}_{i \in I},\left\{u^{i}\right\}_{i \in I}, \pi\right)$. Moreover, if she is risk averse and her endowment is not constant conditional on each payoff relevant state, she will be strictly worse off.

To provide a graphical representation of the proof, consider Figure 8. Even though both $P^{1}$ and $P^{2}$ describe that awareness varies across states $s \in S$, the common generalized prior allows us to enlarge $P^{1}(s)^{S}$ and $P^{2}(s)^{S}$ to $S$ and work only on that state space. In economy $\left(S, P^{1},\left\{\omega^{i}\right\}_{i \in I},\left\{u^{i}\right\}_{i \in I}, \pi\right)$, when $s \in S$ occurs, all agents consider $P^{1}(s)$ to be possible, and in Section 3 we show that the unique equilibrium allocation specifies that each agent consumes her expected endowment given $P^{1}(s)$. Conditional Independence implies that beliefs do not change when we also condition on $\mathcal{E}^{1}(s)$, hence the expected endowment does not change when beliefs are conditioned on $P^{1}(s)^{S} \cap \mathcal{E}^{1}(s)$. The same arguments apply for economy $\left(S, P^{2},\left\{\omega^{i}\right\}_{i \in I},\left\{u^{i}\right\}_{i \in I}, \pi\right)$. Lemma 1 shows that $\mathbb{P}^{1}=\left\{P^{1}(s)^{S} \cap \mathcal{E}_{S}^{1}(s)\right\}_{s \in S}$ and $\mathbb{P}^{2}=\left\{P^{2}(s)^{S} \cap \mathcal{E}_{S}^{2}(s)\right\}_{s \in S}$ are two partitions of $S$, where $\mathbb{P}^{2}$ is finer than $\mathbb{P}^{1}$. In Figure 8, $\mathbb{P}^{1}$ consists of two cells, the first containing the first four states of $S$. Partition $\mathbb{P}^{2}$ is finer than $\mathbb{P}^{1}$ and consists of three cells. This implies that each $i$ 's expected endowment given $P^{1}(s)^{S} \cap \mathcal{E}^{1}(s)$ (for the first four states) is less volatile than $i$ 's expected endowment given $P^{2}(s)^{S} \cap \mathcal{E}^{2}(s)$ (for the first two states) and given $P^{2}\left(s^{\prime \prime}\right)^{S} \cap \mathcal{E}^{2}\left(s^{\prime \prime}\right)$ (for the next two states). For the last three states the expected endowment is the same for both economies. From the law of iterated expectations, the 


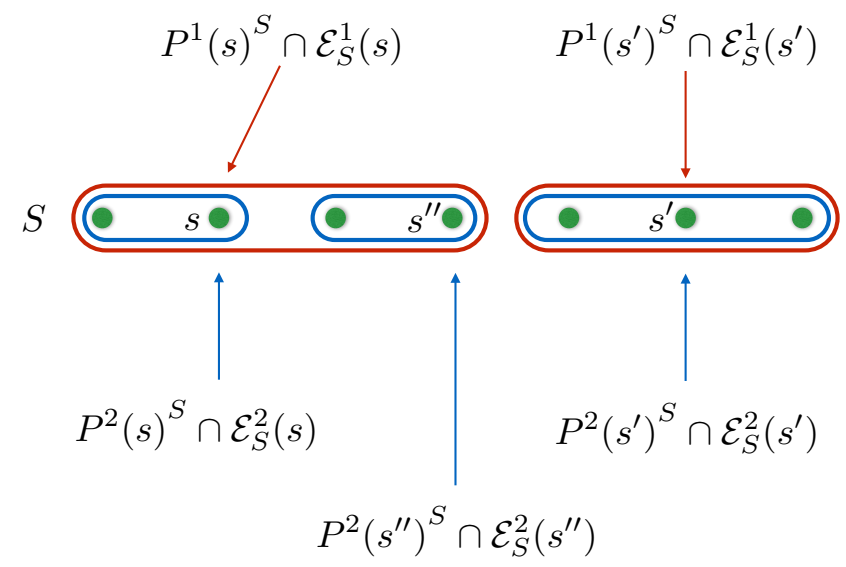

Figure 8: Graphic representation of Theorem 1

ex ante expected values of the random consumptions for the two economies are equal. Because $u^{i}$ is concave, each $i$ weakly prefers the consumption under economy $\left(S, P^{1},\left\{\omega^{i}\right\}_{i \in I},\left\{u^{i}\right\}_{i \in I}, \pi\right)$ than under economy $\left(S, P^{2},\left\{\omega^{i}\right\}_{i \in I},\left\{u^{i}\right\}_{i \in I}, \pi\right)$.

\section{References}

David Andolfatto, Aleksander Berentsen, and Christopher Waller. Optimal disclosure policy and undue diligence. Journal of Economic Theory, 149(C):128-152, 2014.

David Blackwell. Comparison of experiments. In Proceedings of the Second Berkeley Symposium on Mathematical Statistics and Probability, pages 93-102. University of California Press, 1951.

Colin M. Campbell. Blackwell's ordering and public information. Journal of Economic Theory, 114:179-197, 2004.

Eddie Dekel, Bart Lipman, and Aldo Rustichini. Standard state spaces preclude unawareness. Econometrica, 66:159-173, 1998.

Bernhard Eckwert and Itzhak Zilcha. Incomplete risk sharing arrangements and the value of information. Economic Theory, 21(1):43-58, 012003.

Ronald Fagin and Joseph Y. Halpern. Belief, awareness, and limited reasoning. Artificial Intelligence, 34:39-76, 1988.

Spyros Galanis. Syntactic foundations for unawareness of theorems. Theory and Decision, 71 (4):593-614, 2011.

Spyros Galanis. Unawareness of theorems. Economic Theory, 52(1):41-73, 2013. 
Spyros Galanis. The value of information under unawareness. Journal of Economic Theory, 157:384-396, 2015.

John Geanakoplos. Game theory without partitions, and applications to speculation and consensus. Cowles Foundation Discussion Paper, No. 914, 1989.

Piero Gottardi and Rohit Rahi. Value Of Information In Competitive Economies With Incomplete Markets. International Economic Review, 55:57-81, 022014.

Jerry Green. Value of information with sequential futures markets. Econometrica, 49:335-358, 1981.

Jerry Richard Green and Nancy L. Stokey. Two representations of information structures and their comparisons. Technical report, Technical report no. 271 : Economics series: Institute for Mathematical Studies in the Social Sciences, Stanford University, 1978.

Nils Hakansson, Gregory Kunkel, and James Ohlson. Sufficient and necessary conditions for information to have social value in pure exchange. Journal of Finance, 37(5):1169-81, December 1982.

Joseph Y. Halpern. Alternative semantics for unawareness. Games and Economic Behavior, 37: 321-339, 2001.

Joseph Y. Halpern and Leandro Chaves Rêgo. Interactive unawareness revisited. Games and Economic Behavior, 62:232-262, 2008.

Aviad Heifetz, Martin Meier, and Burkhard C. Schipper. Interactive unawareness. Journal of Economic Theory, 130:78-94, 2006.

Aviad Heifetz, Martin Meier, and Burkhard C. Schipper. A canonical model of interactive unawareness. Games and Economic Behavior, 62:232-262, 2008.

Aviad Heifetz, Martin Meier, and Burkhard C. Schipper. Unawareness, beliefs, and speculative trade. Games and Economic Behavior, 77:100-121, 2013.

Jack Hirshleifer. The private and social value of information and the reward to incentive activity. American Economic Review, 61:561-574, 1971.

Edi Karni and Marie-Louise Vierø. "Reverse Bayesianism": A Choice-Based Theory of Growing Awareness. American Economic Review, 103(7):2790-2810, December 2013.

Jean-Jacques Laffont. The Economics of Uncertainty and Information. MIT Press, 1989.

Jing Li. Information structures with unawareness. Journal of Economic Theory, 144:977-993, 2009 .

Sanxi Li, Martin Peitz, and Xiaojian Zhao. Information Disclosure and Consumer Awareness. Technical report, University of Mannheim, Department of Economics, 2014.

Robert McTeer. Mark-to-market accounting: shooting ourselves in the foot. Technical report, Brief Analysis 648, National Center for Policy Analysis, 2009.

Salvatore Modica and Aldo Rustichini. Awareness and partitional information structures. Theory and Decision, 37:107-124, 1994.

Salvatore Modica and Aldo Rustichini. Unawareness and partitional information structures. Games and Economic Behavior, 27:265-298, 1999. 
Stephen Morris. Revising beliefs and knowledge: A generalization of Blackwell's theorem. $C A$ RESS Working Paper \#92-24, 1992.

Stephen Morris and Hyun Song Shin. The rationality and efficacy of decisions under uncertainty and the value of an experiment. Economic Theory, 9:309-324, 1997.

John Quiggin. The value of information and the value of awareness. Theory and Decision, pages $1-19,2015$.

Edward E. Schlee. The value of information in efficient risk-sharing arrangements. American Economic Review, 91(3):509-524, June 2001. 